Artículo de investigación.

Cómo citar: F. Hilarión, E. Bojacá, y D. Bojacá, "Diseño y simulación de un sistema automatizado para producción avícola en la región del Guavio", Inventum, vol. 15 , no. 28 , pp. 9-38. doi: 10.26620/ uniminuto.inventum.15.28.2020.3-32

Editorial: Corporación Universitaria Minuto de Dios - UNIMINUTO.

ISSN: $1909-2520$

eISSN: 2590-8219

Fecha de recibido: 10 de enero del 2020 Fecha de aprobado: 20 de marzo del 2020 Fecha de publicación: 30 de mayo del 2020

Conflicto de intereses: los autores han declarado que no existen intereses en competencia.
Francisco J. Hilarión Novoa francisco.hilarion@unad.edu.co orcid.org/0000-0002-5068-4135 Universidad Nacional Abierta y a Distancia (UNAD). Colombia.

Edgar A. Bojacá Garavito edgar.bojaca@unad.edu.co orcid.org/0000-0002-4604-8849 Universidad Nacional Abierta y a Distancia (UNAD). Colombia.

Diana M. Bojacá Bojacá dmbojacab@unadvirtual.edu.co orcid.org/0000-0002-6413-5997 Universidad Nacional Abierta y a Distancia (UNAD). Colombia.

\section{Diseño y simulación de un sistema automatizado para producción avícola en la región del Guavio}

\author{
Design and simulation \\ of an automated system for poultry \\ production in the Guavio region
}

\author{
Projeto e simulação de um sistema \\ automatizado para produção de aves \\ na região de Guavio
}

\begin{abstract}
Resumen
Los avicultores del municipio de Gachetá, Cundinamarca, Colombia, manifiestan pérdidas en el negocio de crianza/engorde de pollo, tras realizar un proceso de caracterización se encuentra como principales causas las frecuentes interrupciones en el servicio de energía, variación de temperatura y la escasa mano de obra rural, muchos jóvenes se desplazan a la ciudad y abandonan sus labores de producción. Por lo anterior y ante la demanda alimentaria se hace necesario buscar opciones para generar inversión en el municipio con el fin de reactivar el comercio avícola, lo que permitirá la inclusión del desarrollo tecnológico dentro del proceso de crianza/engorde avícola, de allí la necesidad de generar una solución interdisciplinar (electrónica-telecomunicacionesredes y sistemas) para diseñar, simular e implementar en laboratorio computacional un sistema automatizado para producción avícola, alimentado con energía solar fotovoltaica, que permita mejorar los índices de producción y reducir los costos y necesidades de mano de obra. Se describen los procedimientos propuestos para automatizar procesos, se involucran variables como temperatura, humedad, incidencia solar y ventilación, factores determinantes en el proceso de producción avícola en el municipio de Gachetá, mediante el desarrollo de aplicaciones web basadas en MySQL, lenguajes del lado del cliente y del servidor, de la aplicación de Internet de las cosas, simulación y configuración de redes y sistemas. Tras lo anterior se obtiene como resultado el monitoreo y control de variables, almacenamiento de información del proceso y cálculos requeridos para el sistema solar, para analizar las condiciones reales que permitan mejorar toma de decisiones y así asegurar el éxito del negocio.
\end{abstract}

Palabras clave: automatización, avícola, control, desarrollo web, Intenet de las cosas, optimización de producción, rentabilidad.

\begin{abstract}
Poultry farmers in the municipality of Gacheta manifest losses in the business of raising / fattening chicken, after carrying out a characterization process, the main causes are frequent interruptions in the energy service,
\end{abstract}


temperature variation and the scarce rural labor force, many young people move to the city abandoning their production tasks. Due to the above and in view of the food demand, it is necessary to look for options to generate investment in the municipality in order to reactivate the poultry trade allowing the inclusion of technological development within the poultry breeding / fattening process, hence the need to generate a solution interdisciplinary (electronics-telecommunications-networks and systems) to design, simulate and implement in the computer laboratory an automated system for poultry production powered by photovoltaic solar energy, which allows improving production rates, mitigating costs and labor needs. The procedures proposed to automate processes involving variables such as temperature, humidity, solar incidence and ventilation are described as determining factors in the poultry production process in the municipality of Gachetá, through the development of web applications based on MySQL, client-side languages. and the server, the Internet of Things application, simulation and configuration of networks and systems. After the above, the monitoring and control of variables, storage of process information and calculations required for the solar system are obtained as a result, to analyze the real conditions, improving decisionmaking and thus ensuring business success.

Keywords: automation, poultry, control, web development, Internet of things, production optimization, profitability.

\section{Resumo}

Avicultores no município de Gachetá, Cundinamarca, Colômbia, manifestam perdas nos negócios de criação / engorda de frango, após realizar um processo de caracterização é encontrado como principal causa interrupções freqüentes no serviço de energia, variação temperatura e escasso trabalho rural, muitos jovens Deslocam a cidade e abandonam suas tarefas de produção. Para o anterior e antes da demanda de alimentos, é necessário procurar opções para gerar investimentos no município, a fim de reativar a comércio de aves, o que permitirá a inclusão de desenvolvimento tecnológico durante o processo de criação / engorda de aves, daí a necessidade de gerar uma solução interdisciplinar (redes de eletrônica-telecomunicações e sistemas) para projetar, simular e implementar em laboratório um sistema automatizado para produção de aves, alimentado por energia solar fotovoltaica, o que permite melhorar a taxas de produção e reduzir custos e necessidades de mão-de-obra. Os procedimentos propostos para automatizar processos são descritos, variáveis como temperatura, umidade, incidência solar e ventilação, fatores determinantes no processo de produção avícola no município de Gachetá, através do desenvolvimento de aplicações web baseados no MySQL, linguagens do lado do cliente e do servidor, Aplicação Internet of things, simulação e configuração de rede e sistemas. Após o exposto, o monitoramento e controle variável, armazenamento de informações do processo e cálculos necessários para o sistema solar, para analisar as condições reais que permitem melhorar a tomada de decisões e, assim, garantir o sucesso de negócios.

Palavras-chave: automação, aves, controle, desenvolvimento web, Internet das coisas, otimização da produção, lucratividade. 


\section{INTRODUCCIÓN}

A partir del levantamiento de información a través de fuentes primarias, se encuentra que los avicultores de la región del Guavio se han visto afectados por los continuos cortes de energía eléctrica, necesaria para la correcta iluminación y nutrición de las aves, las variaciones de temperatura y la escasa mano de obra. De acuerdo con el "Estudio de factibilidad para el diseño de un sistema automatizado para producción avícola alimentado con energía solar" adelantado por los investigadores del semillero Searpro UNAD en el municipio de Gachetá, se determinó que las condiciones de temperatura, humedad e incidencia solar son adecuadas y no se requiere calefacción.

También cabe resaltar las amenazas identificadas por la Universidad de Los Andes para el sector avícola relacionadas con la escasa tecnología, mano de obra sin calificar, falta de capacitación técnica, impactos por fenómenos naturales, bajos volúmenes de producción y carencia de impulso de actividades especializadas, así como las preocupantes cifras reveladas por la Federación Nacional de Avicultores, las cuales solamente toman el cuenta la producción del municipio de Fómeque con un PIB avícola de menos de 25.000 millones de pesos, el resto de la región se considera irrelevante.

Por lo anterior se considera necesario diseñar y simular computacionalmente un sistema automatizado para producción avícola alimentado con energía solar fotovoltaica, de esta forma se pretende monitorear la temperatura, humedad, iluminación, y a su vez automatizar las cortinas de control de temperatura, la iluminación, el flujo de agua y comida requerido para la alimentación de las aves, con una fuente de alimentación alternativa como lo es la energía solar fotovoltaica.

El proceso de diseño y simulación de las variables a automatizar comenzará, con la ayuda de tarjetas de desarrollo Arduino, con los módulos de comunicación, actuadores y sensórica necesarios para el correcto monitoreo, procesamiento y control de variables. A su vez se integrará la programación de un aplicativo por medio de lenguajes de programación, que pueden ser tipo web o móvil que permita establecer un puente e interacción del usuario con el sistema.

La importancia del proyecto radica en generar una solución interdisciplinar para diseñar, simular e implementar, en laboratorio computacional, un sistema automatizado para producción avícola alimentado con energía solar fotovoltaica, que permita reactivar la economía avícola, mejorar los índices de producción, mitigar costos y necesidades de mano de obra, lo que ayudará en la toma de decisiones y el éxito en el negocio.

\section{PROBLEMÁTICA}

De acuerdo con los resultados del estudio de factibilidad para el diseño de un sistema automatizado para producción avícola alimentado con energía solar, los avicultores del municipio de Gachetá se han visto afectados en el proceso de crianza y engorde de pollos, debido a pérdidas generadas por fallas en el servicio eléctrico, y estabilización de temperatura, "ya que esta varía en horas de la tarde y noche". Otros factores que han afectado el proceso son: suministro inadecuado de alimento, falta de control de flujo de agua, manejo inadecuado de gases, entre otros. Tras realizar el estudio de factibilidad donde se evidenciaron aspectos fundamentales para el uso de la energía solar fotovoltaica se evidenció una viabilidad para su uso en el municipio de Gachetá, y tras la elaboración del diseño y simulación de un sistema automatizado que responde a las necesidades de producción del galpón, se identificaron los mecanismos para optimizar el proceso de crianza y engorde de pollos.

De acuerdo con la Universidad de los Andes, mediante el diagnóstico productivo agroambiental para la jurisdicción del Guavio, se evidencia en una matriz DOFA, amenazas importantes para el sector avícola, tales como: baja tecnología, mano de obra sin calificar, falta de capacitación técnica, impactos por fenómenos naturales, bajos volúmenes de producción y carencia de impulso de actividades especializadas. Así mismo se encuentra una población de apenas 2500 aves en Gachetá, muy por debajo de poblaciones como Fómeque con 7800 aves y Gachalá con 6600 aves. Por lo anterior, Gachetá evidencia una baja producción a pesar de contar con las condiciones óptimas para producción avícola sostenible.

La caracterización avícola para el Departamento de Cundinamarca desarrollada por Fenavi, muestra unas cifras muy preocupantes, en las cuales el mercado avícola para la región del Guavio y más concretamente para Gachetá es irrelevante a pesar de contar con condiciones óptimas para este proceso, donde la pauta la marca Fusagasugá con un PIB avícola de 75.000 millones de pesos, Guaduas con más de 25.000 millones de pesos, Arbeláez con más de 25.000 millones de pesos y Fómeque con menos de 25.000 millones de pesos. 
Por otra parte, conforme al diagnóstico productivo agroambiental de la Universidad de los Andes, se evidencia que en la región del Guavio existe una baja capacitación técnica, poca o nula tecnología y deficiente mano de obra. Lo que hace necesario generar espacios donde la tecnología y más concretamente los procesos de automatización avícola, que son un factor determinante para suplir la carencia de mano de obra calificada, satisfacer la demanda de carne de pollo, que según Fenavi va en aumento, y a su vez constituye una oportunidad de desarrollo para el municipio de Gachetá. Finalmente, conforme lo indica la Revista de Medicina Veterinaria de la Universidad Militar, se establece que las pérdidas económicas por enfermedades infeccionas y otros agentes son inestimables por el productor y no se encuentra ningún informe estadístico oficial que mida estos aspectos en el municipio de Gachetá. [1]

Por lo cual se plantea como pregunta orientadora la siguiente: ¿Cómo el diseño, simulación e implementación computarizada de un sistema automatizado para producción avícola alimentado con energía solar fotovoltaica puede mitigar los factores de riesgo de pérdida en la crianza/engorde de pollos y generar mayor rentabilidad?

\section{ENERGÍA SOLAR EN GACHETÁ Y SUS POSIBLES APORTES AL DESARROLLO ECONÓMICO DEL MUNICIPIO}

\section{A. Caracterización del municipio}

La producción de energía es hoy en día una de las mayores exigencias que tiene una región, su influencia directa en la economía y en el desarrollo generan gran interés, pues su uso eficiente a bajo costo permitirá tener mejores resultados y mayor competitividad en el mundo actual, mejorar la calidad de vida los habitantes y generar un mayor respeto por la naturaleza.

Con la hidroeléctrica del Guavio esta región ha generado millones de watios que han llegado al hogar de miles de usuarios, lo que les ha permitido satisfacer sus necesidades energéticas. Una gran inversión que ha representado millones de ganancias a empresas públicas y privadas, sin embargo es importante pensar ¿qué otra alternativa energética puede satisfacer las necesidades de los pobladores del municipio a bajo costo y con buena calidad?

La energía solar es entonces una alternativa que puede ser analizada como pequeña generadora de energía alternativa limpia, en especial para aquellos procesos agropecuarios que requieren pocas cantidades de energía, energía que no puede ser interrumpida por la exigencia del proceso. Tras generar análisis del entorno del municipio de Gachetá y con ayuda del atlas solar, se relaciona el siguiente estudio de incidencia solar para el municipio. El proceso de medición realizado por el equipo investigador con ayuda del atlas solar del Ideam, mes a mes arroja los siguientes datos:

\begin{tabular}{|l|c|c|}
\hline \multicolumn{1}{|c|}{ Mes } & $\begin{array}{c}\text { Medida mínima } \\
\text { en } \mathrm{kw} / \mathrm{m}^{2}\end{array}$ & $\begin{array}{c}\text { Medida máxima } \\
\text { en } \mathrm{kw} / \mathrm{m}^{2}\end{array}$ \\
\hline Enero & 4,5 & 5 \\
\hline Febrero & 4,5 & 5 \\
\hline Marzo & 4 & 4,5 \\
\hline Abril & 3,5 & 4 \\
\hline Mayo & 3,5 & 4 \\
\hline Junio & 3,5 & 4 \\
\hline Julio & 3,5 & 4 \\
\hline Agosto & 3,5 & 4 \\
\hline Septiembre & 4 & 4,5 \\
\hline Octubre & 4 & 4,5 \\
\hline Noviembre & 4 & 4,5 \\
\hline Diciembre & 4 & 4,5 \\
\hline
\end{tabular}

Tabla I. Radiación solar mes a mes en Gachetá Fuente: los autores.

Como se evidencia en el análisis realizado anteriormente, los meses de mayor radiación solar en el municipio de Gachetá, son enero y febrero, con medidas desde 4,5 a $5 \mathrm{kw} / \mathrm{m}^{2}$, en los meses de abril hasta agosto la incidencia se mantiene con valores que oscilan entre los 3,5 y $4 \mathrm{kw} / \mathrm{m}^{2}$, mientras que en los meses de septiembre a diciembre los valores oscilan entre los 4 y $4,5 \mathrm{kw} / \mathrm{m}^{2}$, lo anterior significa que en el municipio de Gachetá se generan en promedio $4,12 \mathrm{kw} / \mathrm{m}^{2}$.[2]. Por lo anterior se deben determinar los parámetros y posibles usos con el fin de generar procesos energéticos donde la energía solar pueda responder de forma eficiente con las necesidades definidas dentro de procesos agropecuarios. Antes de fijar un proceso específico o en la gama de procesos donde la energía solar puede ser utilizada se debe tener en cuenta que los equipos encargados de generar energía solar se alimentan de la captación de radiación solar para funcionar. Mientras más radiación solar sea captada por un equipo, mayor será su eficiencia. Se analiza entonces el potencial económico del municipio, el cual cuenta con grandez extensiones de tierra sin 
explotar, en su mayoría dedicados a la ganadería, avicultura y pequeñas plantaciones de caña de azúcar, café y gulupa.

El sector agrícola es el principal potencial de desarrollo, sin embargo, se encuentra abandonado en su gran mayoría, ya que los habitantes no encuentran atractivos los negocios de producción alimentaria, debido principalmente a los costos de mano de obra, poca asistencia técnica, pocos canales de venta, entre otros que permitan tener un mercado organizado. Se encuentran en las diferentes veredas cultivos de café, maíz, yuca, arracacha, papa, fríjol, guayaba, caña panelera, mora y algunos pequeños cultivos de hortalizas [3]. El sector agropecuario no muestra una uniformidad en su producción, por el contrario, en las diferentes veredas se encuentra en pequeñas cantidades cultivos de café, frijol, caña de azúcar, mora, lulo, entre otros, sin embargo, a pesar de su diversidad, muchos de estos cultivos se realizan de forma transitoria y en la mayoría de los casos no se da continuidad a la producción o se cultiva para el autoconsumo, por otro lado, existen pequeños sectores encargados de la producción avícola, porcícola, ganadera y de piscicultura. Haciendo énfasis en el desarrollo de la producción avícola se ha encontrado que para el caso del municipio de Gachetá se cuenta con un gran potencial para aumentar en gran medida el sector de la producción avícola, tras realizar un estudio de factibilidad de producción avícola en Gachetá se encontró que las temperaturas oscilan entre los $18^{\circ} \mathrm{C}$ y $19.5^{\circ} \mathrm{C}$, las máximas temperaturas encontradas varían entre $21^{\circ} \mathrm{C}$ y $24,3^{\circ} \mathrm{C}$ en la parte alta del municipio, sobre los 2400 m.s.n.m., y $24,6^{\circ} \mathrm{C}$ y $27,9^{\circ} \mathrm{C}$ en la parte baja, en alturas que oscilan entre $1.800 \mathrm{y}$ 2.000 m.s.n.m. Teniendo en cuenta los parámetros establecidos, el grupo investigador realizó el proceso de medición en el galpón y encontró que la temperatura mínima de crianza para los pollos debe ser de mínimo $19^{\circ} \mathrm{C}$ y una máxima de $27^{\circ} \mathrm{C}$ para la temperatura del galpón, lo anterior significa que según las condiciones climatológicas el municipio de Gachetá cumple con los requisitos[3].

\section{B. Diseño y simulación}

Los avicultores de la región del Guavio se han visto afectados en el proceso de crianza y engorde de pollos, debido a pérdidas generadas por fallas en el servicio eléctrico, temperaturas variables en horas de la tarde y noche, por lo que se hace necesario preguntar ¿Cómo el diseño de un sistema automatizado para producción avícola alimentado con energía solar fotovoltaica puede mitigar los factores de riesgo de pérdida en la crianza/engorde de pollos y generar mayor rentabilidad en menor tiempo?

Buscando optimizar los resultados de dicha producción se plantea entonces el diseño y simulación de un sistema automatizado para producción avícola alimentado con energía solar fotovoltaica como alternativa para el desarrollo de los campesinos productores de pollo apoyados por el uso de energías limpias.

\section{DETERMINAR LAS ESPECIFICACIONES DEL SISTEMA A AUTOMATIZAR}

Tras la validación del equipo investigador se han determinado las siguientes condiciones del sistema a automatizar:

\section{A. Ventilación - Calidad del aire}

Es importante que los pollos siempre tengan niveles adecuados de oxígeno y mínimos niveles de $\mathrm{CO}_{2}, \mathrm{CO}, \mathrm{NH}_{3}$ y polvo, ya que su calidad incidirá en forma directa en el proceso de crianza y engorde lo contrario traerá como consecuencia elevados niveles de amoniaco, dióxido de carbono y humedad, que a su vez pueden desencadenar ascitis y enfermedades crónicas del tracto respiratorio de los pollos dentro del galpón lo que se traduce en pérdidas económicas. [4] Por otro lado, los niveles de amonio deben evaluarse al nivel de las aves y deben mantenerse todo el tiempo bajo 10 ppm, un mal manejo de este nivel traerá como consecuencia quemaduras de patas, ampollas en la pechuga, lesiones de ojo, lesiones de piel, baja uniformidad, bajo peso corporal, mayor susceptibilidad a enfermedades y ceguera. Los niveles de calidad del aire se definen de acuerdo con los siguientes datos:

\begin{tabular}{|l|c|}
\hline \multicolumn{1}{|c|}{ Oxigeno \% } & $\varnothing 19,6$ \\
\hline Dióxido de Carbono $\left(\mathrm{CO}_{2}\right)$ & $<0,3 \% / 3,000 \mathrm{ppm}$ \\
\hline Monóxido de Carbono & $<10 \mathrm{ppm}$ \\
\hline Amoniaco & $<10 \mathrm{ppm}$ \\
\hline Humedad relativa & $45 \mathrm{a} 65 \%$ \\
\hline Polvo respirable & $<3,4 \mathrm{mg} / \mathrm{m}^{3}$ \\
\hline
\end{tabular}

Tabla II. Niveles de calidad del aire

Fuente: [4] 
Los pollitos son muy susceptibles al aire, pequeñas corrientes del orden de los $0,5 \mathrm{~m} / \mathrm{s}(100 \mathrm{ft} / \mathrm{min})$ pueden generar efecto de enfriamiento en especial en aquellos recién nacidos por lo que los dispositivos destinados a la ventilación deben apuntar en otras direcciones y conservar un nivel mínimo de generación de corrientes de viento [5].

\section{B. Manejo por semana}

Dentro del galpón se deben garantizar elementos fundamentales y básicos que garanticen un producto de calidad, dando un manejo específico por semana.

\section{Primera semana}

- Revisar la temperatura diariamente, esta debe oscilar entre 30 a $32{ }^{\circ} \mathrm{C}$ [6], por lo que se propone el diseño de un sistema de apertura y cierre de cortinas de acuerdo a temperatura.

- El primer día suministrar en el agua de bebida suero casero supervisado con sensores y electroválvulas.

- Suministrar la totalidad de alimento diaria sobre las bandejas racionalmente (varias veces al día), el sistema supervisará la cantidad de comida y acorde a ello generará el suministro de forma automática.

- Realizar el pesaje semanal e ingresar la información en una base de datos para ver la trazabilidad de la crianza y engorde.

- Analizar el consumo de alimento de acuerdo con el alimento suministrado.

- Garantizar la iluminación nocturna. Esta es una buena alternativa para alimentar al pollo. Ya que la temperatura será más fresca, y el animal estará más confortable y dispuesto para comer, también se debe garantizar al menos una hora de oscuridad para acostumbrar a los animales a situaciones de oscuridad y mitigar pérdidas por falta de iluminación [7].

\section{Segunda semana}

La temperatura que se manejará dentro de esta semana será de 26 a $28^{\circ} \mathrm{C}$.
- Cuadrar densidad y alturas de bebederos y comederos, los bebederos a la altura de la espalda y los comederos a la altura de la pechuga.

- Nivelar los bebederos automáticos a la altura de la pechuga.

- Realizar el pesaje semanal e ingresar la información en una base de datos para ver la trazabilidad de la crianza y engorde.

- Registrar mortalidad y sacrificio e ingresar la información en una base de datos para ver la trazabilidad.

\section{Tercera semana}

- La temperatura debe estar entre 20 y $24^{\circ} \mathrm{C}$.

- Nivelar los bebederos automáticos a la altura de la pechuga [6].

- Se llenan los comederos de concentrado el sistema supervisara la cantidad de comida y acorde a ello genera el suministro de forma automática.

- Realizar el pesaje semanal e ingresar la información en una base de datos para ver la trazabilidad de la crianza y engorde.

- Registrar mortalidad y sacrificio e ingresar la información en una base de datos para ver la trazabilidad.

\section{Cuarta semana a séptima}

- Verificar temperatura ambiente diariamente.

- Realizar el pesaje 2 veces por semana e ingresar la información en una base de datos para ver la trazabilidad de la crianza y engorde.

\section{Requerimientos para automatizar}

Por lo anterior se puede determinar que el sistema a automatizar debe responder a los siguientes requerimientos:

- Control de amoniaco dentro del galpón.

- Control de temperatura en la primera semana, entre 30 a $32{ }^{\circ} \mathrm{C}$. 
- Control de temperatura en la segunda semana, será de 26 a $28^{\circ} \mathrm{C}$.

- Control tercera semana temperatura, debe estar entre 20 y $24^{\circ} \mathrm{C}$.

- Control de calidad del agua y suministro de alimento.

- Base de datos para registro del pesaje semanal.

- Control de iluminación donde se garantice una hora de oscuridad al día.
- Análisis estadístico del consumo de alimentos.

- Base de datos del peso del animal.

- Base de datos para registrar la mortalidad del animal por enfermedad o sacrificio.

- Altura de los bebederos.

- Sistema de desinfección con luz ultravioleta, o similares.
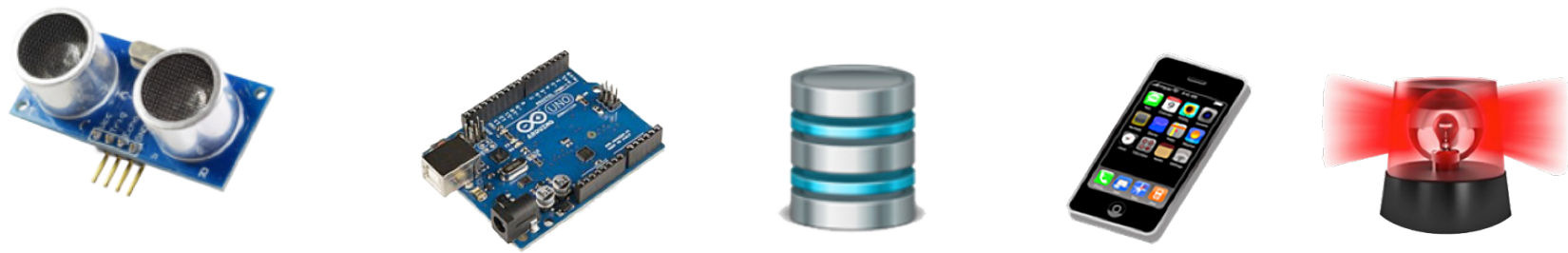

Sensor de nivel de agua Sensor de cantidad de alimento Sensor de iluminación Sensor de temperatura Sensor de humedad Sensor de calidad de aire Sensor de calidad de agua
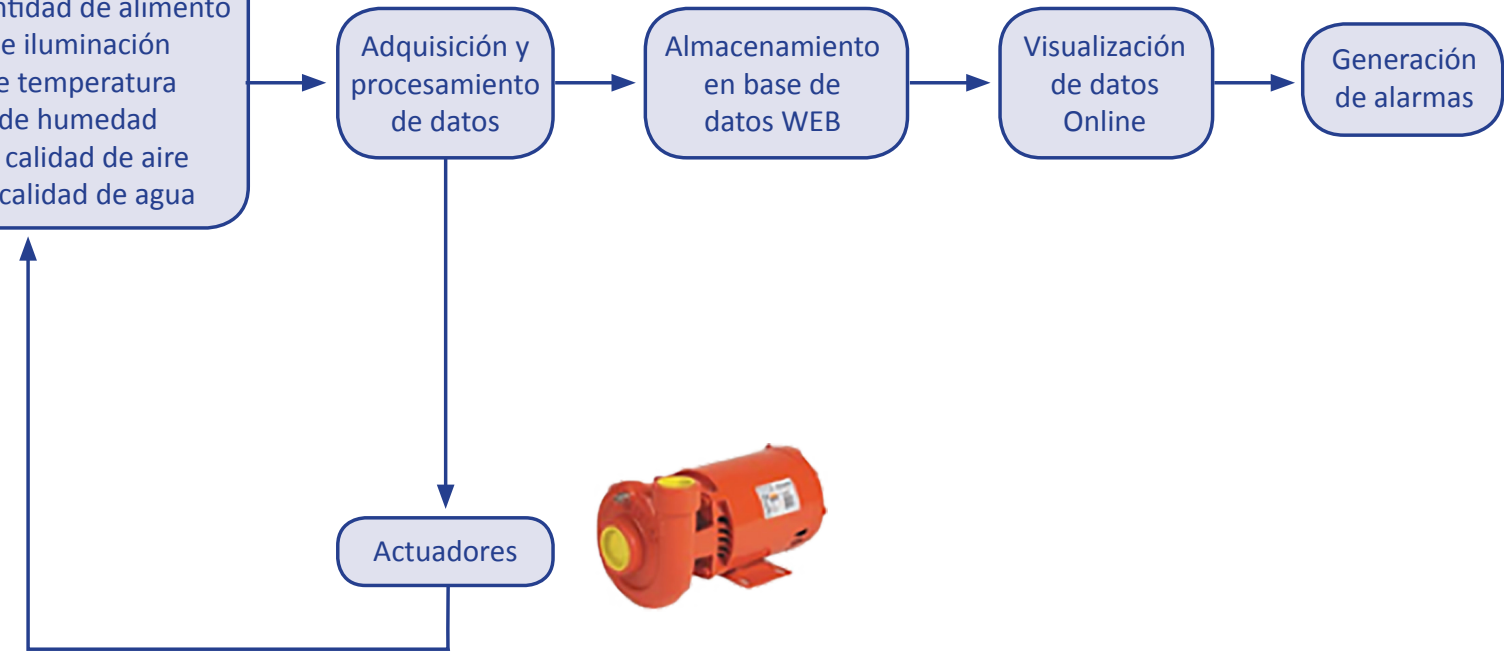

Figura 1. Diagrama de bloques, sistema de información y control.

Fuente: los autores. 


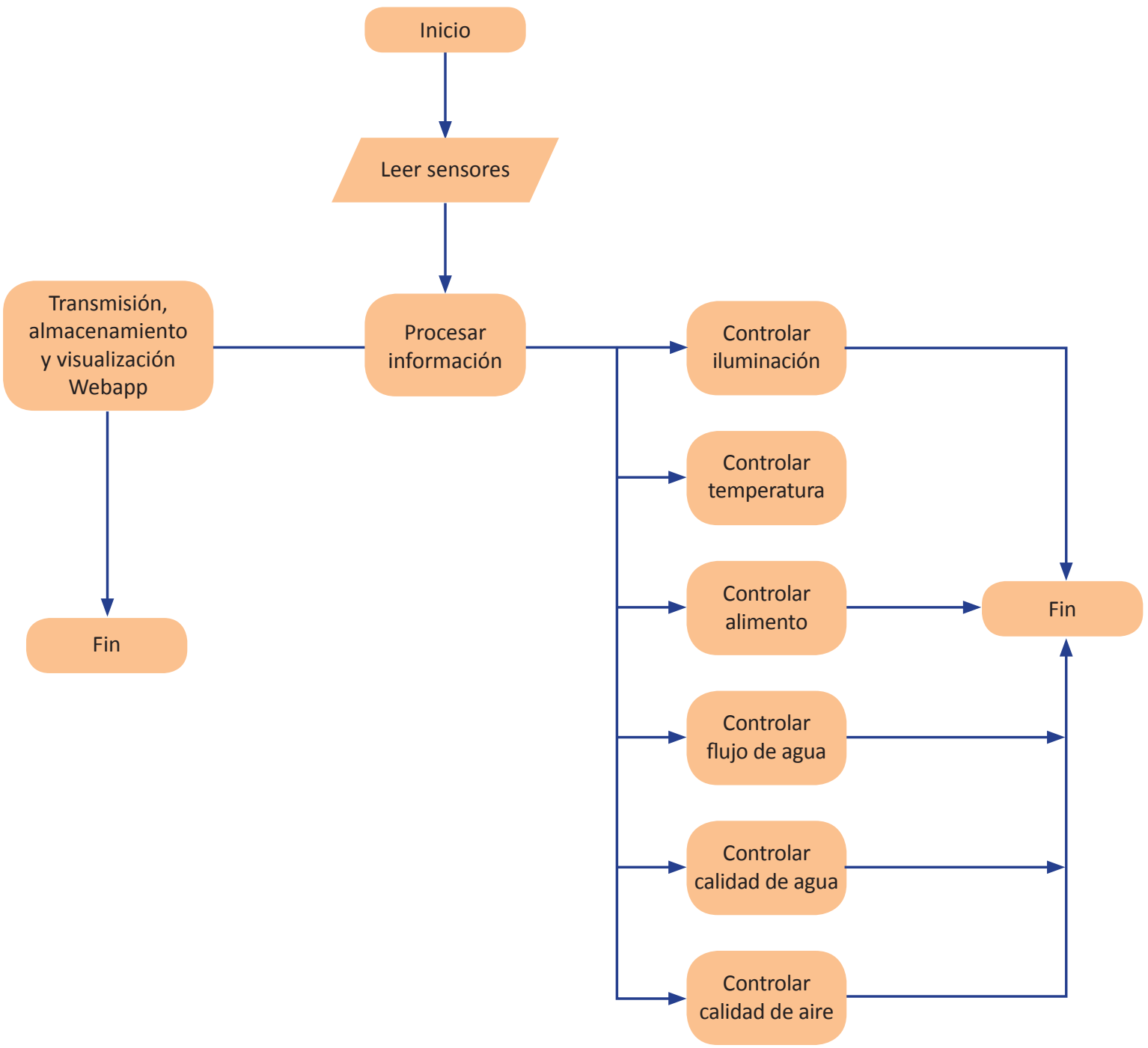

Figura 1. Algoritmo sistema de información y control.

Fuente: los autores.

\section{DIAGRAMA DE BLOQUES Y ALGORITMOS}

Teniendo en cuenta las variables que se han definido para automatizar dentro del diseño y simulación del sistema automatizado para producción avícola alimentado con energía solar fotovoltaica se han definido los siguientes procesos.

\section{A. Diagrama de bloques:}

Sistema de información y control

Para este caso se propone que los sensores definidos para cada uno de los procesos de monitoreo envíen información a través de una placa
Arduino, que ejecuta un proceso de conversión análogo digital con el fin de ingresar la información al sistema, dicha información se almacena en la base de datos, la cual permitirá visualización de la información mediante la consulta en un servidor web que cuenta con un entorno desarrollado en PHP y HTML, de las señales detectadas por los sensores y de acuerdo a parámetros definidos se activan actuadores para abrir o cerrar cortinas, suplir de alimento o agua a los pollos o activar alarmas, las cuales se pueden visualizar en el aplicativo apoyado de indicaciones con iluminación led o pantallas LCD en las situaciones críticas. 


\section{B. Control de amoniaco}

El proceso de control de amoniaco se encarga de validar la calidad del aire. Las señales son procesadas generando la conversión análogo digital, dicha información es procesada por la tarjeta Arduino que transfiere la información al servidor web, una vez el amoniaco es detectado se genera una alerta, la cual debe activar los actuadores encargados de la ventilación, así como la apertura de cortinas [8].

Para lo anterior se propone el uso del sensor MQ135, este es un sensor analógico por lo que es fácil de implementar con cualquier microcontrolador, además es electroquímico y varía su resistencia cuando se expone a determinados gases, internamente posee un calentador encargado de aumentar la temperatura interna y con esto el sensor pueda reaccionar con los gases y provocar un cambio en el valor de la resistencia.

El MQ-135 es un sensor de gases peligrosos utilizado para el control de la calidad del aire y es adecuado para la detección de $\mathrm{NH}_{3}$ (amoníaco), NOx, alcohol, benceno, humo, $\mathrm{CO}_{2}$, etc.
Este sensor no proporciona valores absolutos, sino que simplemente proporciona una salida analógica que debe ser monitoreada y es comparada con los valores de umbral [9]. Para el caso de nuestro diseño los valores en unidades correspondientes a la medición del gas, se necesita escalar el valor leído, el problema de esto es que la relación entre la lectura analógica y el valor real no es lineal. Por lo que necesitamos estimar la curva que nos da el datasheet.

\section{Curva característica de sensibilidad}

La figura 3 muestra el típico características de sensibilidad de MQ-135 para varios gases.

Temp: 20

Humedad: $65 \%$

Concentración de 02 21\%

$\mathrm{RL}=20 \mathrm{k} \Omega$

Ro: resistencia del sensor a $100 \mathrm{ppm}$ de $\mathrm{NH}_{3}$ en el aire limpio.

Rs: Resistencia del sensor en varias concentraciones de gases.

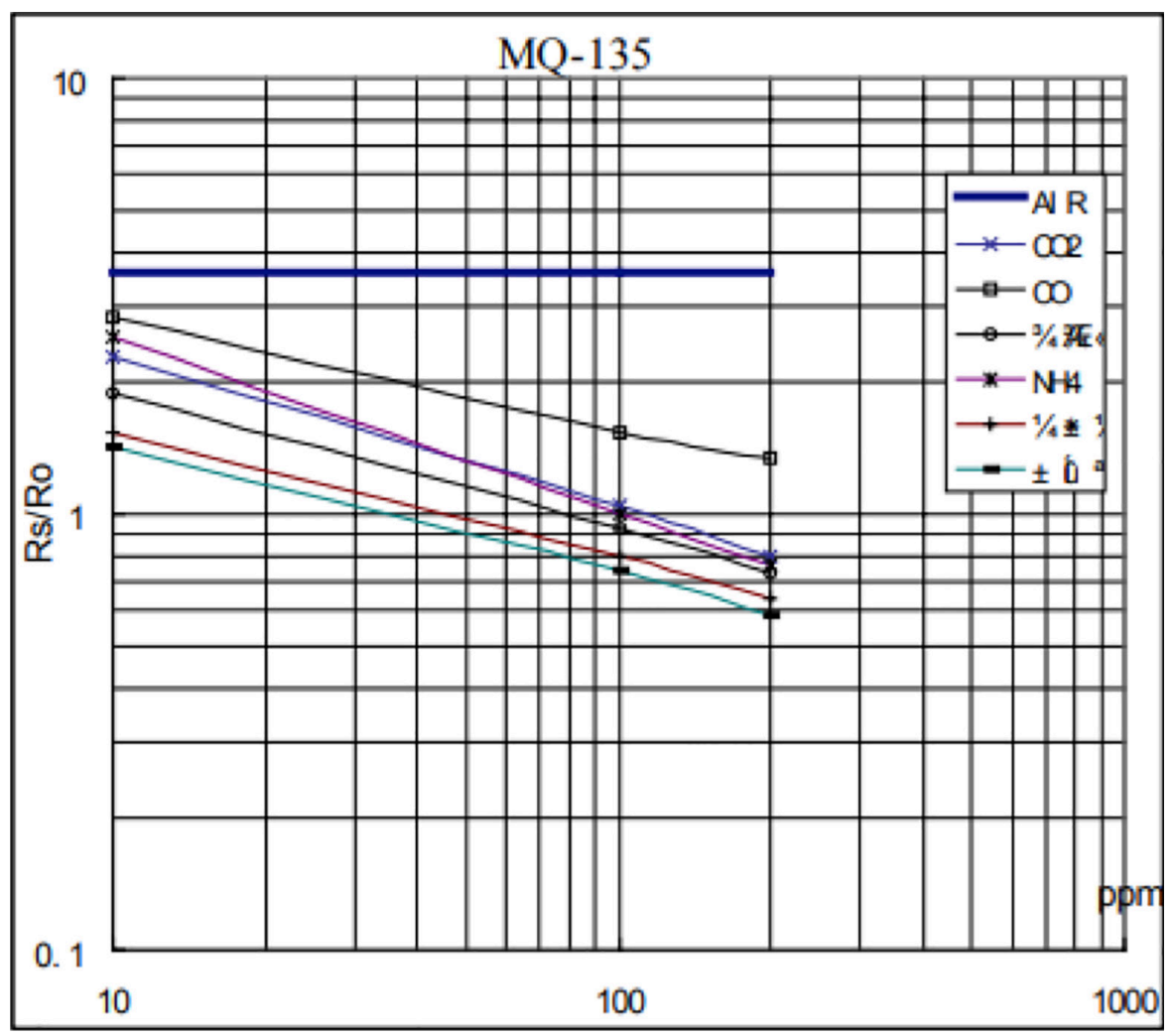

Figura 3. Características de sensibilidad de MQ-135 para varios gases. Fuente: [10]. 
La figura 4 muestra la dependencia típica del MQ135 sobre temperatura y humedad.

Ro: resistencia del sensor a $100 \mathrm{ppm}$ de $\mathrm{NH}_{3}$ en el aire a $33 \%$ HR y 20 grados.

Rs: resistencia del sensor a $100 \mathrm{ppm}$ de $\mathrm{NH}_{3}$ a diferentes temperaturas y humedades.

\section{Control de temperatura}

El proceso de supervisión de temperatura se realiza utilizando el sensor DTH11, el cual genera una señal digital que ingresa a la tarjeta Arduino, de acuerdo al tiempo de crianza en semanas se definen condiciones para la apertura y cierre de cortinas, las cuales son las principales responsables de garantizar condiciones de temperatura optima dentro del galpón, la información de variación de temperatura es almacenada en una base de datos de un servidor web, dicha información permite además un análisis histórico de temperatura en la zona que puede ser usada en la planeación de futuros proyectos.

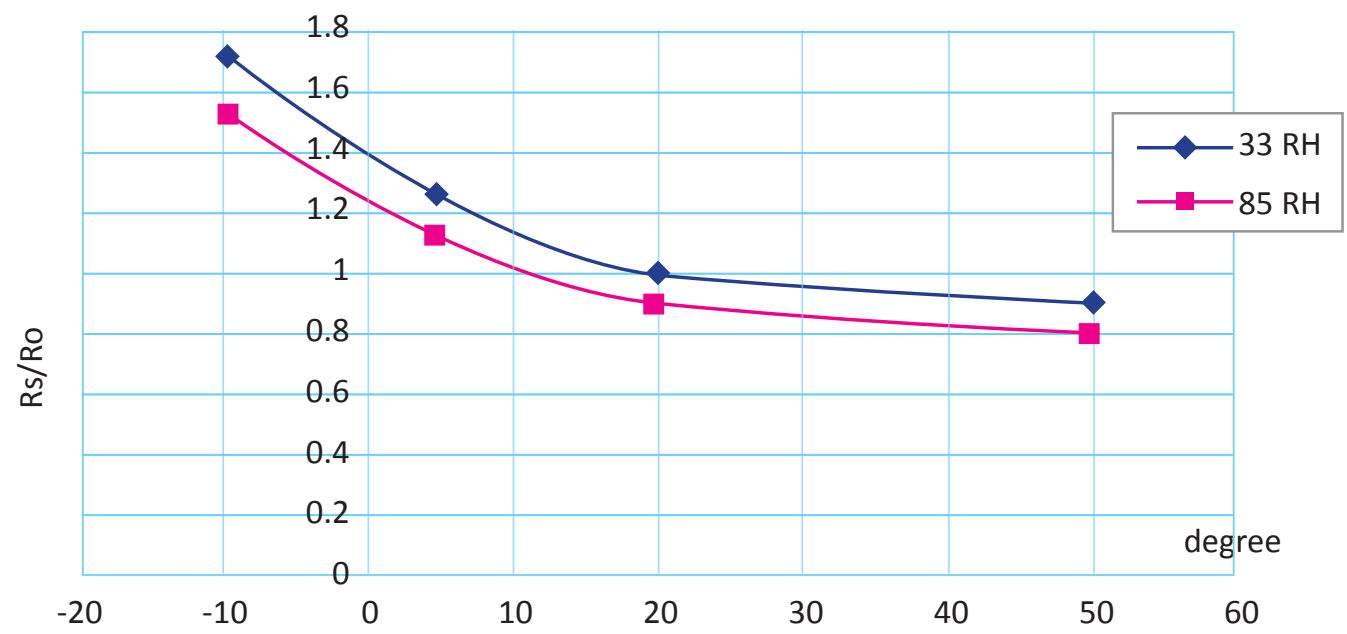

Figura 4. Dependencia típica sobre temperatura y humedad.

Fuente: [10].

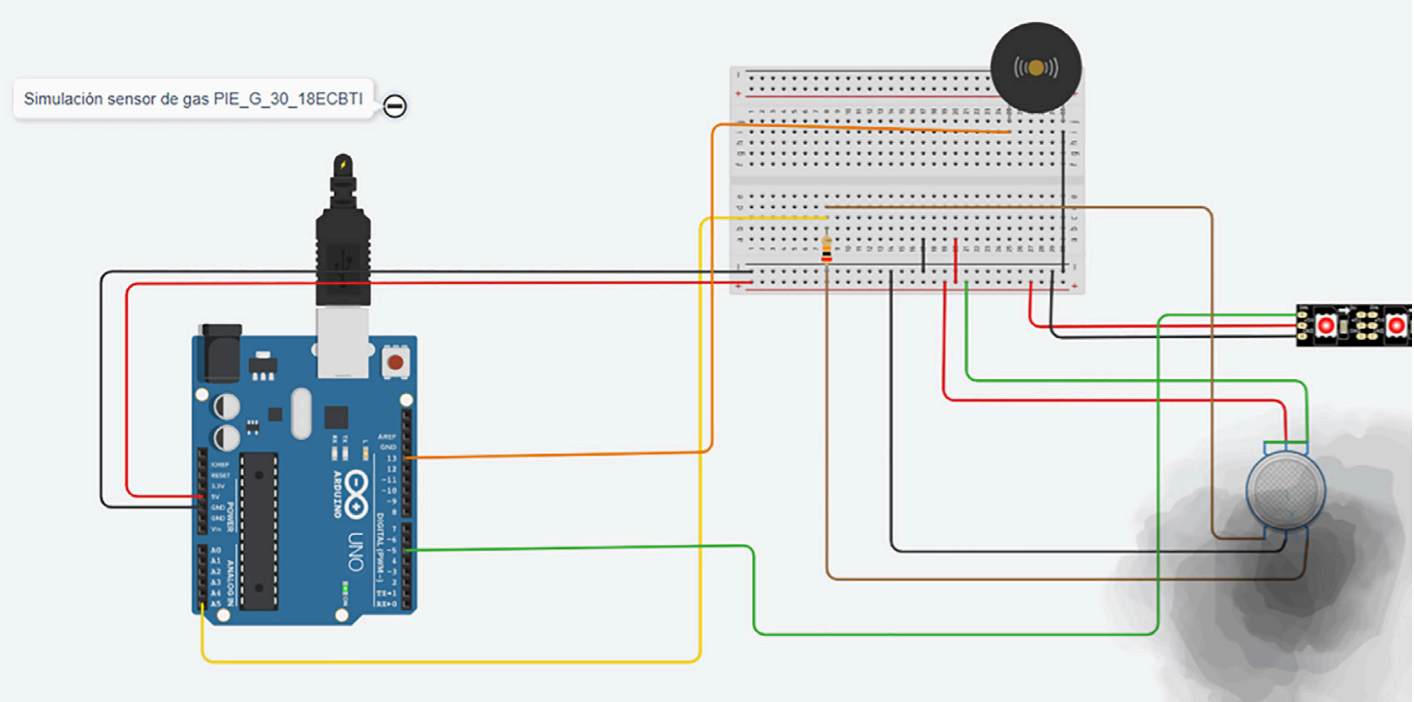

Figura 5. Circuito básico sensor MQ-135.

Fuente: [10]. 


\section{¿Cómo transmite los datos el DHT11?}

El sensor se conecta a un pin digital, se trata de un dispositivo analógico. Dentro del propio dispositivo se hace la conversión entre analógico y digital [11].

Por lo tanto, se parte de una señal analógica que luego es convertida en formato digital y se enviará al microcontrolador. La trama de datos es de 40 bits correspondiente a la información de humedad y temperatura del DHT11 [12].

El primer grupo de 8-bit es la parte entera de la humedad y el segundo grupo la parte decimal. Lo mismo ocurre con el tercer y cuarto grupo, la parte entera de la temperatura y la parte decimal.

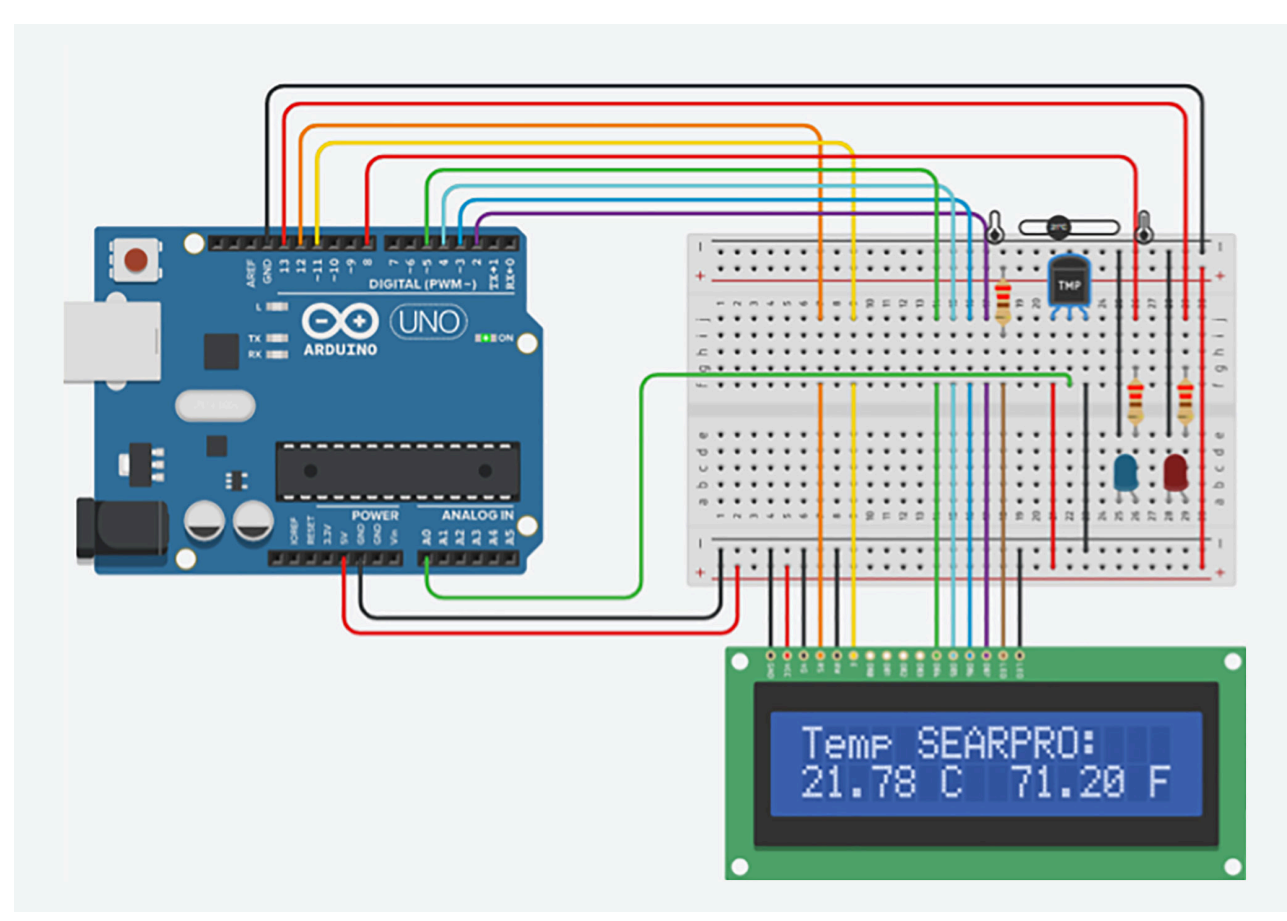

Figura 6. Monitoreo de temperatura con pantalla LCD.

Fuente: los autores.

Por último, los bits de paridad para confirmar que no hay datos corruptos [13]. Estos bits de paridad lo único que hacen es asegurar que la información es correcta, al sumar los 4 primeros grupos de 8-bit.

Esta suma debe ser igual a los bits de paridad. Si se observa la imagen anterior y se suman los bits, se comprueba que todo está correcto.

$$
\begin{aligned}
& 00110101+00000000+ \\
& 00011000+00000000=01001101 .
\end{aligned}
$$

El diagrama de flujo de la siguiente página muestra el proceso de verificación por semanas, de acuerdo con los requerimientos establecidos.

\section{Suministro de alimento y agua (T2)}

El flujo de alimento y agua es quizá uno de los factores de mayor importancia en el proceso de crianza, el diseño de la automatización establece como fundamental censar la presencia de alimentos y agua en los comederos y bebederos disponibles para los pollos, básicamente tras realizar el proceso de verificación por parte de los sensores se procesa la información, la cual es almacenada en base de datos del servidor y a su vez se genera el proceso de activación de actuadores que suministran el alimento o agua [14]. 


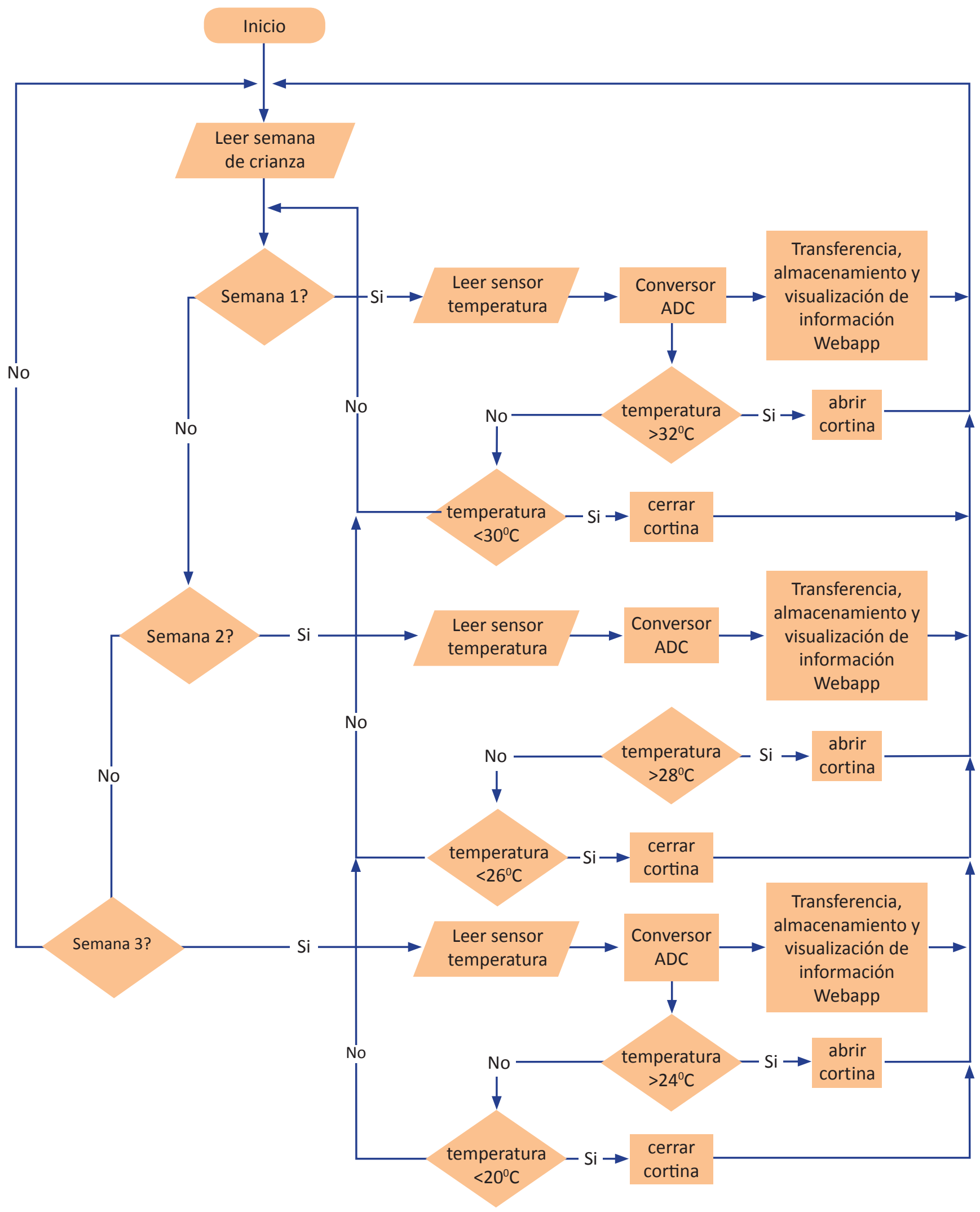

Figura 7. Proceso de verificación por semanas.

Fuente: los autores. 


\section{E. Suministro de alimento}
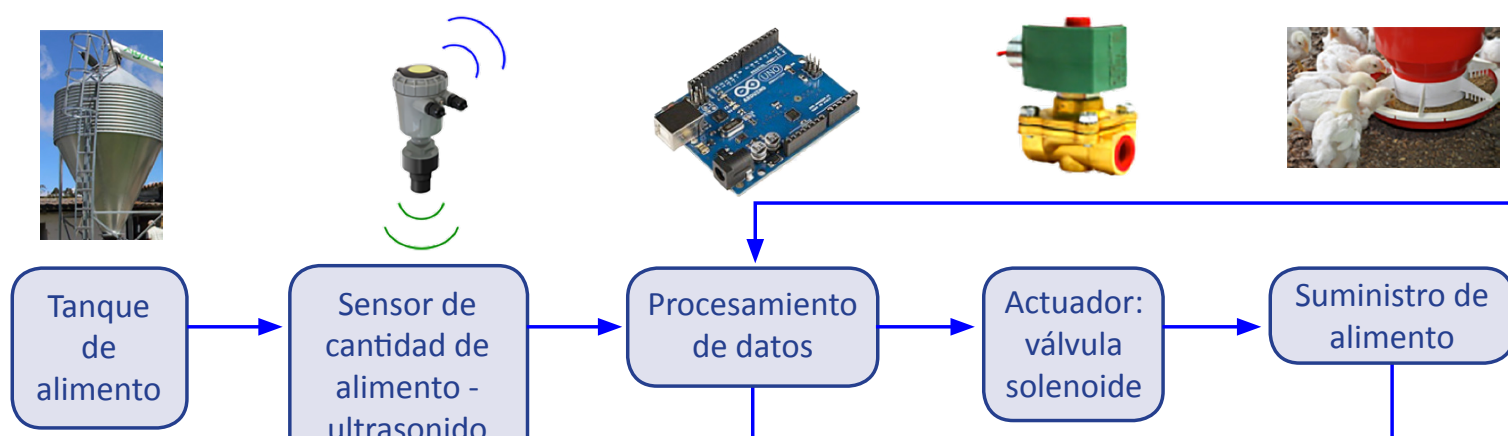

Sensor de cantidad de alimento ultrasonido

Diagrama de bloques suministro de alimento
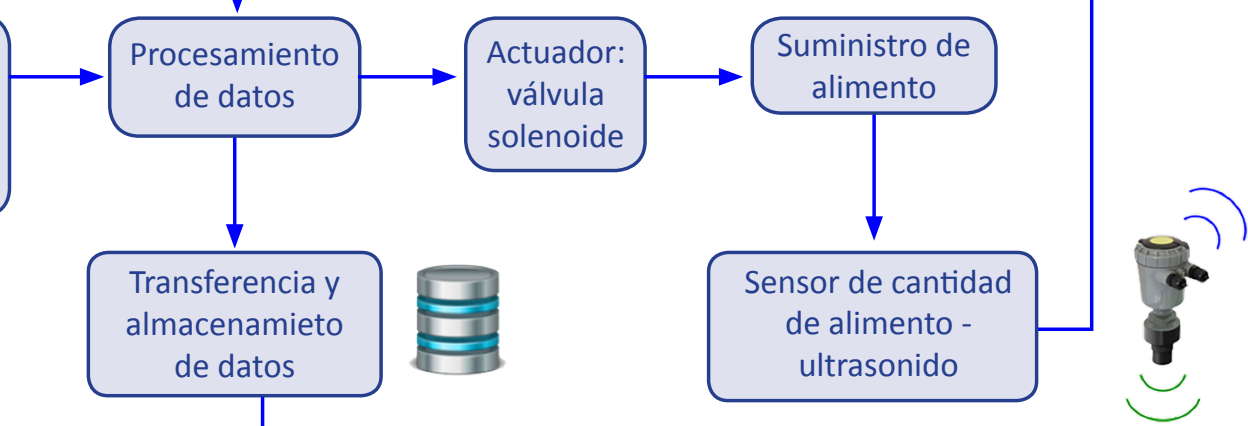

Figura 8. Diagramas de bloques suministro de alimento.

Fuente: los autores.

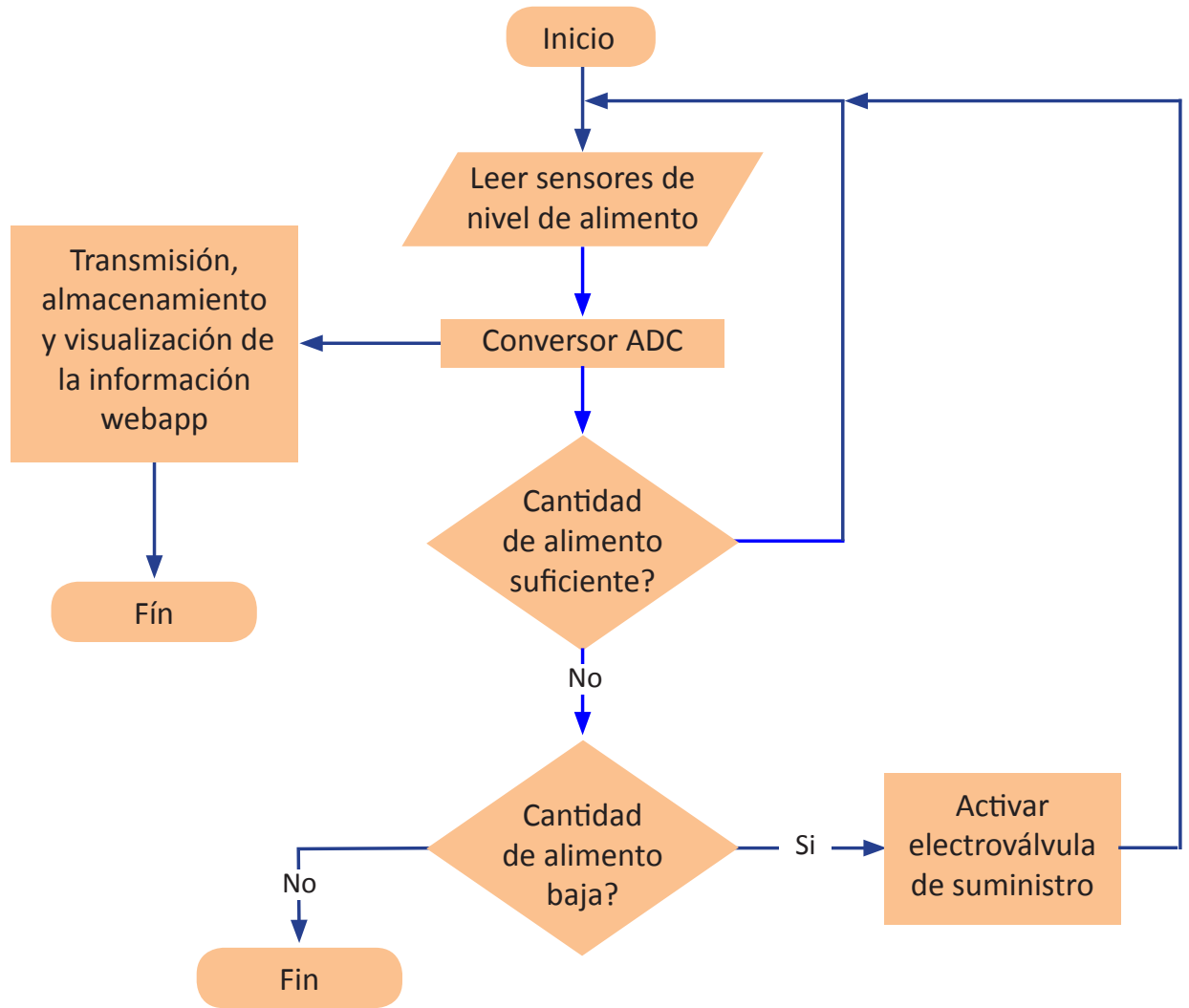

Figura 8. Diagramas de bloques suministro de alimento.

Fuente: los autores. 


\section{F. Suministro de agua}
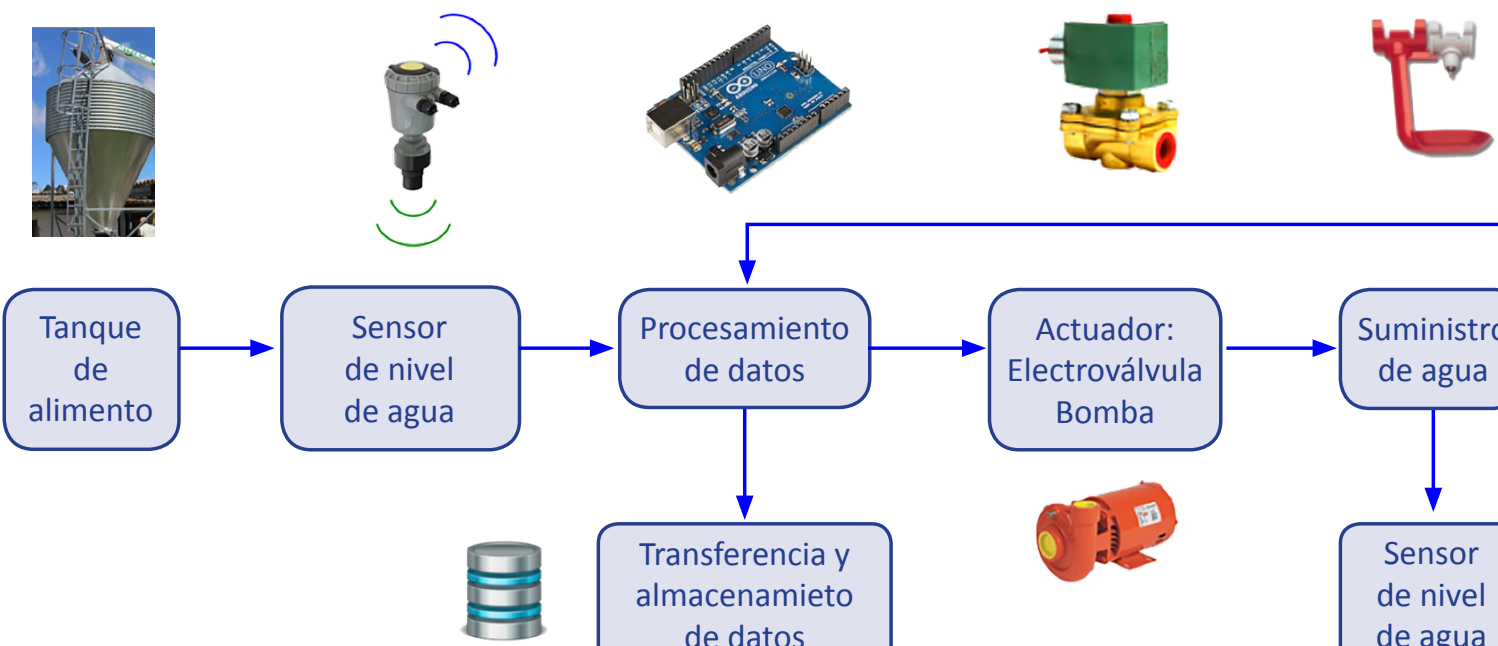

Diagrama de bloques suministro de agua

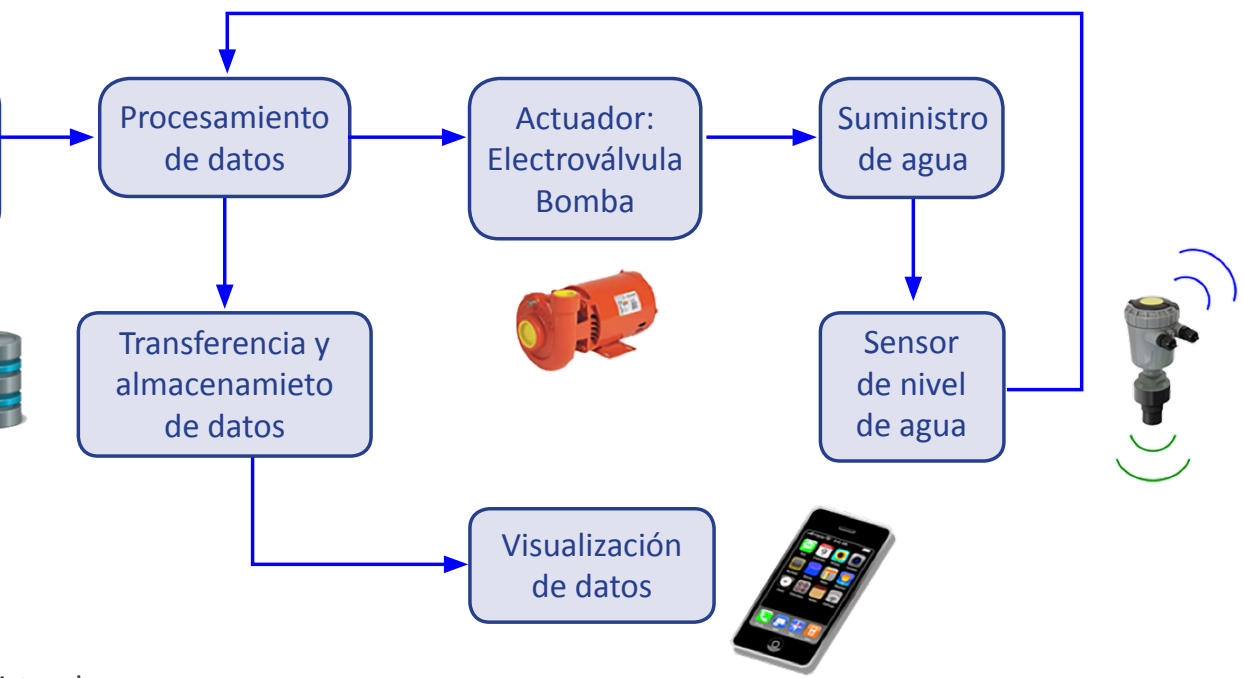

Figura 10. Diagramas de bloques suministro de agua

Fuente: los autores.

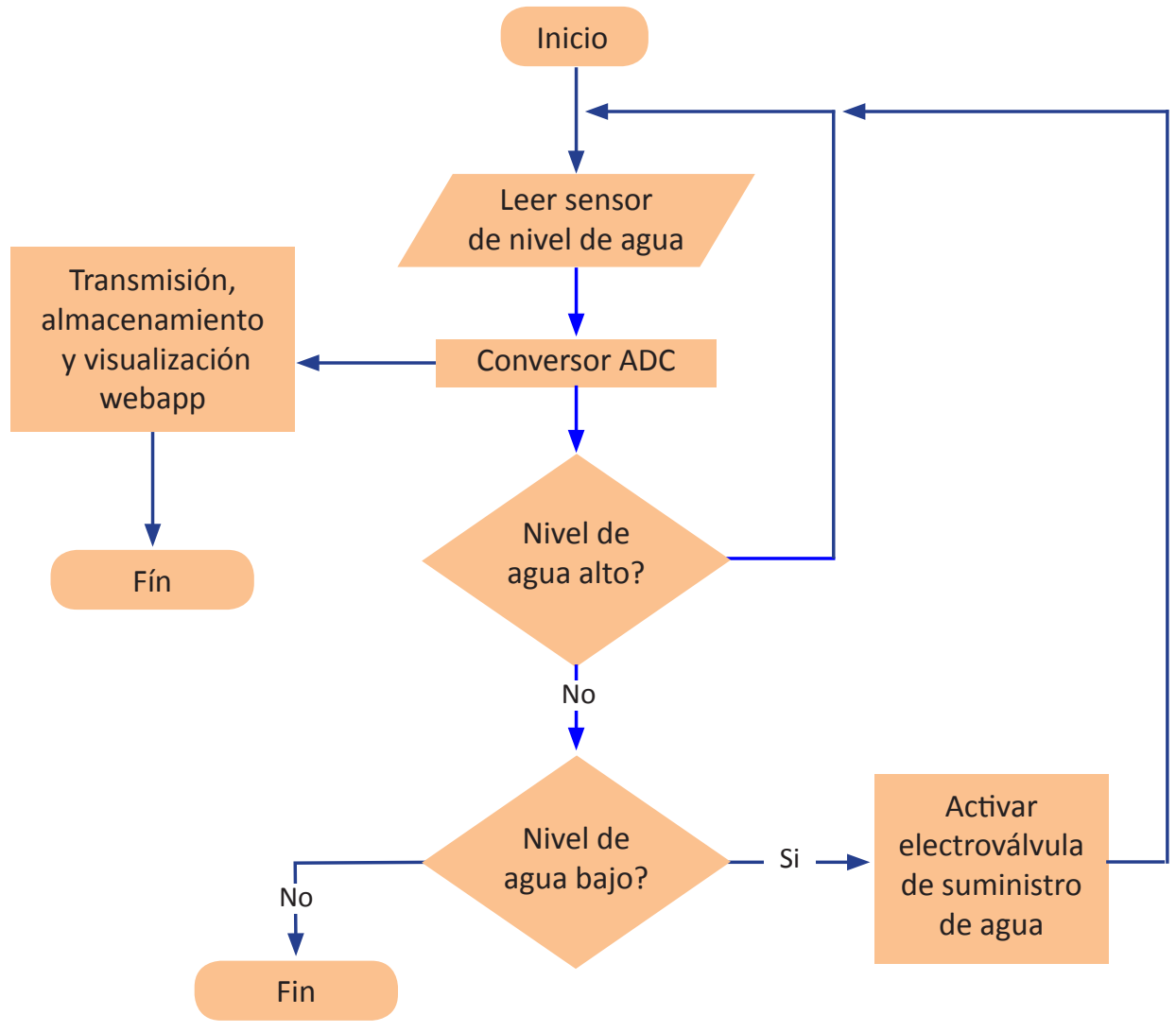

Figura 11. Diagrama de flujo suministro de agua Fuente: los autores. 


\section{G. Control de iluminación}

El control de la iluminación permitirá un mayor desarrollo del animal en menor tiempo, lo anterior debido a que esto motiva al animal a comer con mayor frecuencia. De acuerdo a los hallazgos definidos por el equipo investigador es recomendable que la iluminación se suspenda solo durante una hora en un periodo de 24 horas, se propone mantener la iluminación con led por el bajo consumo energético, utilizando un control de lampara led PWM generado por Arduino y acorde con la edad del animal [15].

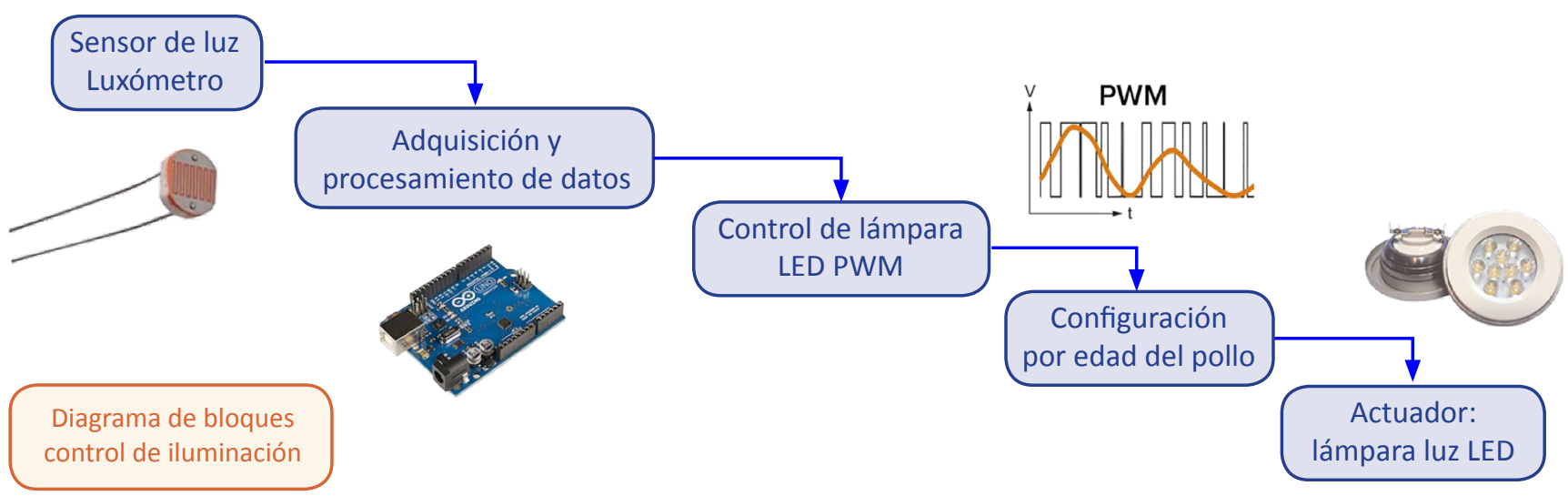

Figura 12. Diagramas de bloques control de iluminación.

Fuente: los autores.

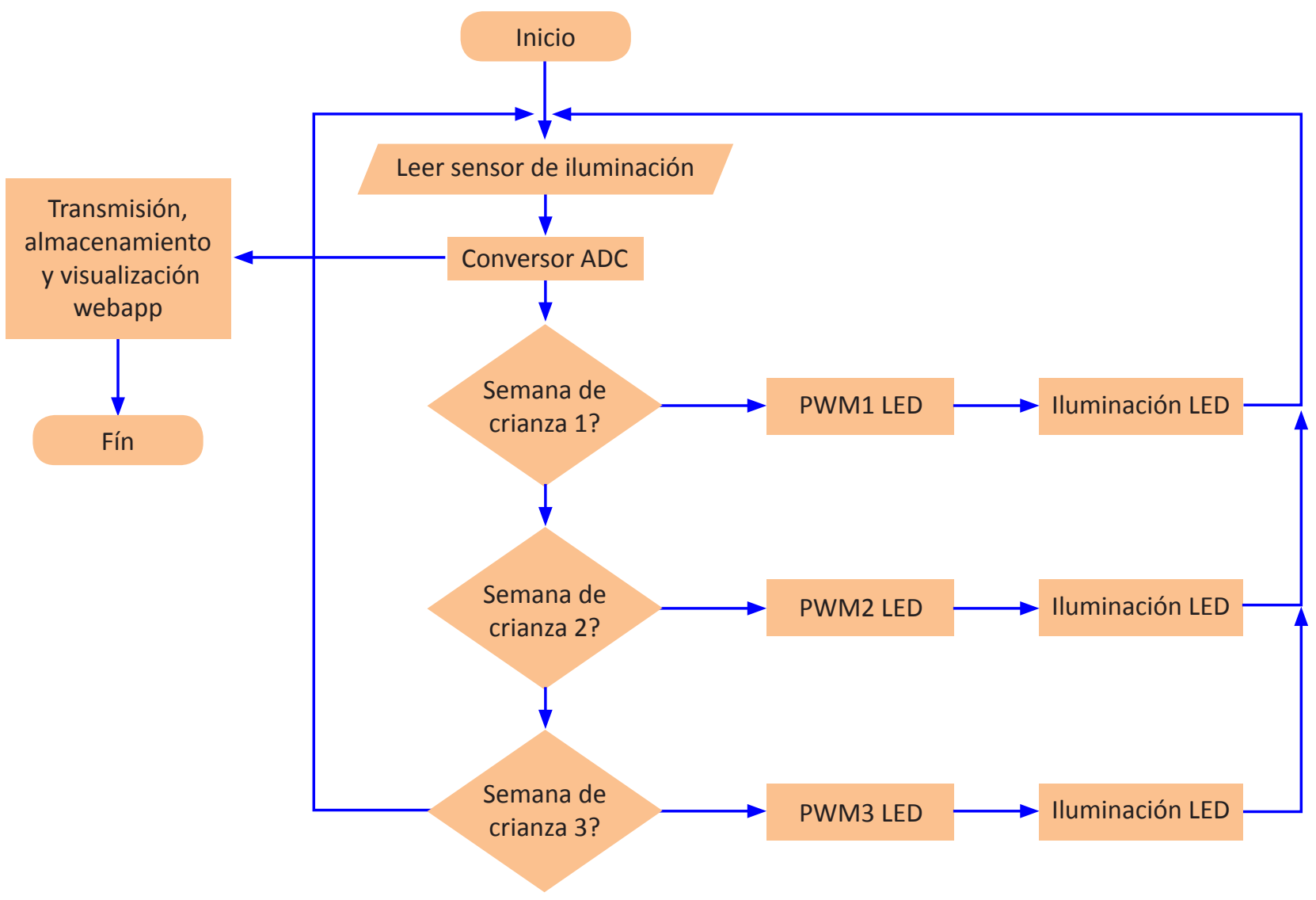

Figura 13. Diagramas de flujo control de iluminación.

Fuente: los autores. 


\section{PLATAFORMA INTERNET DE LAS COSAS}

Es importante detallar cada bloque del sistema que se desarrolla. La sensórica del prototipo está soportada en sensores de temperatura y humedad relativa, que entregan los datos en forma digital, la adquisición y procesamiento de datos se constituye en tarjetas de desarrollo Arduino y NodeMCU, el desarrollo web integra PHP del lado del servidor, MySQL como sistema de gestión de bases de datos y CSS, HTML, Bootstrap, del lado del cliente como lenguajes que permiten la visualización de los datos y la interfaz entre el usuario y el sistema. El bloque de actuadores se basa en solenoides y electroválvulas para control de fluidos y solidos previamente acoplados por una interfaz de potencia.
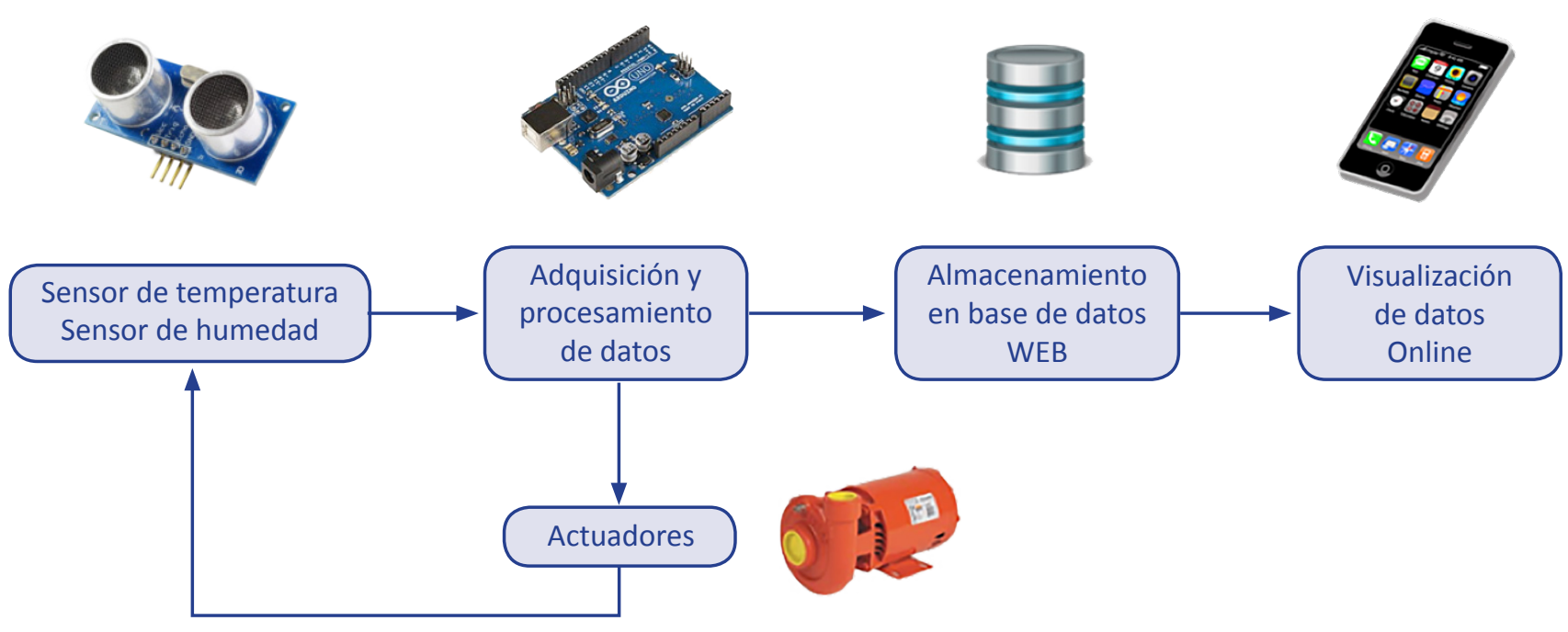

Figura 13. Diagrama de bloques sistema.

Fuente: los autores.

\section{ADQUISICIÓN DE DATOS}

La adquisición de datos es aquel proceso que permite recoger valores de variables, en este caso temperatura y humedad relativa [16].

Para adquirir datos del medio ambiente se requiere un sensor que tenga contacto con el medio y un sistema de adquisición de datos encargado de adecuar y acondicionar la señal adquirida y entregarla en un formato preciso y seguro para su lectura por medio de un microcontrolador, a través de un convertidor análogo a digital, dependiendo del tipo de variable a medir [17].

En este caso puntual se hace uso del sensor DHT11, el cual permite una lectura de temperatura y humedad relativa de forma digital, precisa y segura, lista para que se pueda interpretar con un microcontrolador y generar acciones de control, almacenamiento o comunicación con otros módulos y sistemas [18].

\section{PROCESAMIENTO DE DATOS}

El procesamiento de datos es una etapa en la que básicamente se interpretan los datos que provienen de un sensor con su señal previamente acondicionada [19].

Para el procesamiento de datos, generalmente se emplea un microcontrolador, el cual integra una memoria RAM para almacenar información de trabajo, una CPU que se encarga de las tareas de procesamiento central, una memoria ROM para almacenar el código que se encarga de comandar las tareas del microcontrolador, unos puertos de entrada y salida, con el fin de recibir los valores de los sensores, efectuar tareas de comunicación serial y conexión de otros periféricos para el comando de actuadores [20].

En este caso particular se elige un microcontrolador destinado a Internet de las cosas, básicamente es una tarjeta de desarrollo con base en un NodeMCU 
que integra un módulo ESP8266 para comunicaciones Wifi, tiene memoria RAM, memoria ROM, puertos de entrada/salida y es de fácil manejo, ya que se puede alimentar con 5VDC y anclar a una placa de prototipos [21].

Para este caso el sensor DHT11 se ha instalado en el pin D4 de la tarjeta de desarrollo NodeMCU, y los respectivos pines de alimentación se alimentan directamente de la tarjeta a 3.3VDC como se aprecia en la figura 15.

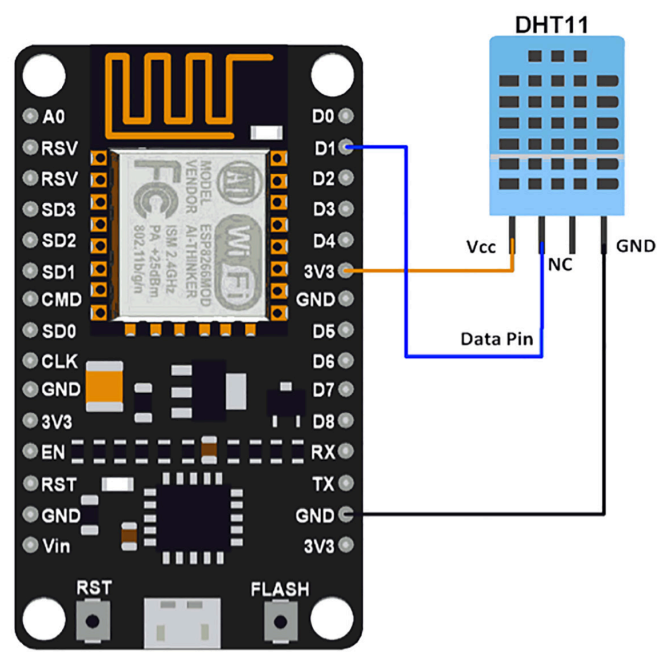

Figura 15. Conexión Node MCU y sensor Fuente: [22].

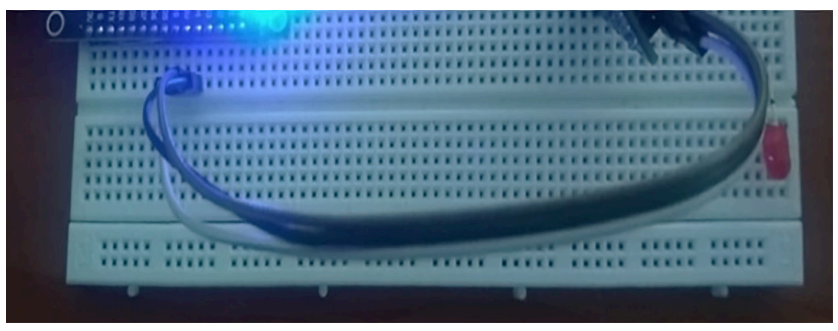

Figura 16. Prototipo Node MCU y sensor.

Fuente: los autores.

En cuanto a la programación de la tarjeta de desarrollo deben incluirse las librerías para el manejo del sensor DHT11 y para el manejo de la tarjeta NodeMCU ESP8266, las cuales se llaman: <ESP8266WiFi.h y <DHT.h>, dichas librerías evitan tener que realizar implementación de comandos AT para el correcto funcionamiento de la tarjeta de desarrollo y permiten trabajar directamente con las variables de temperatura y humedad del módulo DHT11 [23].

\section{\#include $\langle$ ESP8266WiFi.h〉 \#include "DHT.h"}

Figura 17. Librerías Arduino.

Fuente: los autores.

Posteriormente ha de configurarse el nombre de la red Wifi y la contraseña de esta red, con el objetivo de conectar a la red la tarjeta de desarrollo. Para que la tarjeta pueda conectarse a un servidor, donde se aloja la base de datos y el desarrollo web, hay que especificar por medio de una constante de tipo "char" la dirección del servidor. [24].

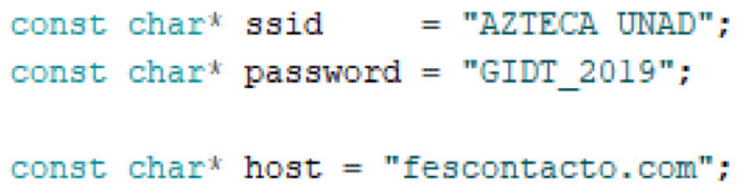

Figura 18. Variables definidas.

Fuente: los autores.

En cuanto al proceso de conexión, se realiza la inicialización del módulo y del sensor por vía serial a 9600 baudios, se indica la orden de conectarse a la red Wifi especificada por medio del identificador SSID y la contraseña determinada [25].

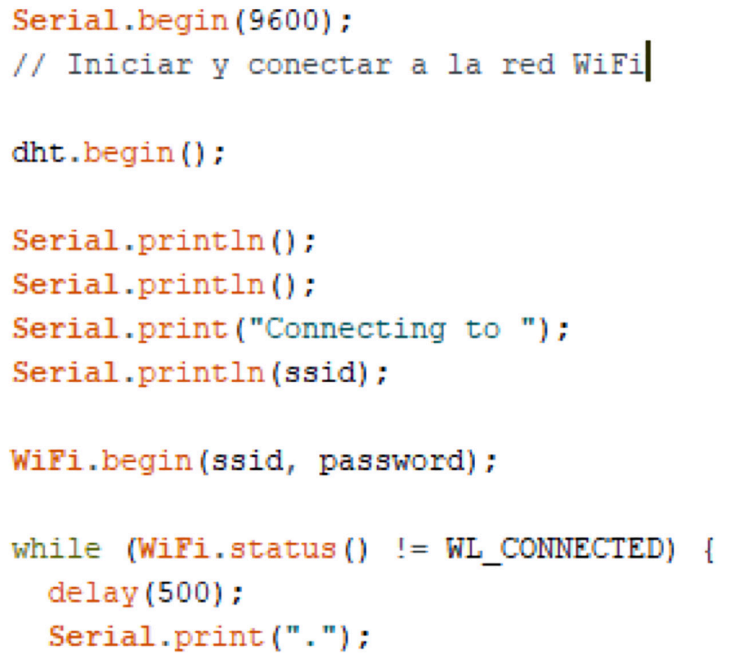

Fig. 19. Códigos conexión Wifi.

Fuente: los autores. 
Posteriormente se deben crear dos variables de tipo flotante para la temperatura y la humedad relativa, se leen y se guardan para ser enviadas al servidor que las gestionará [26].

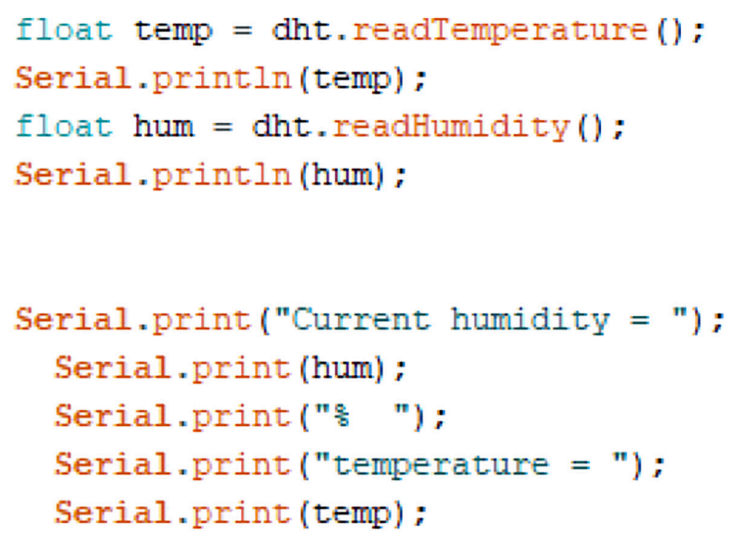

Fig. 20. Captura de variables.

Fuente: los autores.

\section{ENVÍO DE DATOS A LA NUBE}

Para que los datos adquiridos y procesados mediante la tarjeta de desarrollo puedan migrarse a la nube, se hace necesario un servidor y un módulo de comunicación, que para este caso se realiza mediante el módulo ESP8266 y el microcontrolador NodeMCU [27].

Se configura el cliente Wifi mediante la librería, que permite el acceso al servidor especificado, mediante el uso del módulo serial del microcontrolador y se accede por medio del puerto 80 [28].

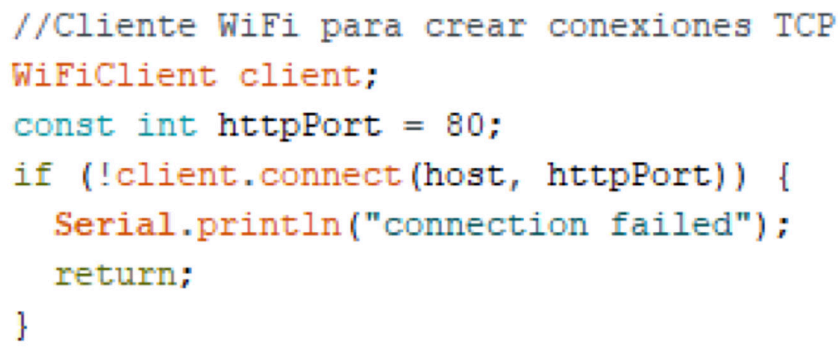

Fig. 21. Código cliente Wifi.

Fuente: los autores.

En el servidor existe un archivo en lenguaje PHP que permite capturar los datos de las variables y almacenarlos en una base de datos para luego gestionarlos. Se debe crear una solicitud con los datos para direccionarlos al programa en lenguaje PHP, para ello se realiza una programación de la tarjeta de desarrollo que cumpla con esos parámetros.

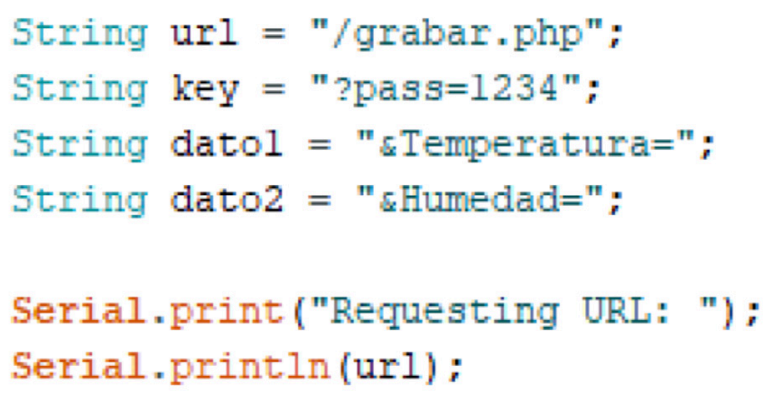

Fig. 22. Solicitud URL.

Fuente: los autores.

Posteriormente se realiza el envío de la petición al servidor, básicamente se envían los datos de las variables de temperatura y humedad por medio del método GET, para proteger el aplicativo web de ataques se integra una contraseña para que no se reciba cualquier dato que pudiera ser inyectado por terceros [29].

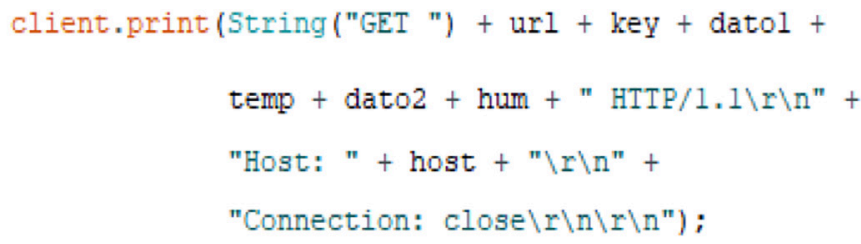

Fig. 23. Petición GET.

Fuente: los autores.

Para finalizar el envío de datos a la nube se cierra la conexión con el servidor para evitar el uso de recursos innecesarios, y pasados 10 segundos se vuelve a realizar todo el proceso, es decir se crea un bucle con el fin de realimentar y actualizar los datos en el servidor [30].

\section{GESTIÓN DE DATOS}

La gestión de bases de datos permite registro, actualización, consulta y eliminación de información de tablas que han sido creadas mediante modelos de entidad relación [31]. 
La herramienta de modelado de datos conocida como entidad relación, permite mostrar las entidades más relevantes de un conjunto de datos, sus interrelaciones y propiedades específicas [32].

En cuanto al modelado para el almacenamiento de datos de variables de temperatura y humedad se tiene en cuenta por defecto tres campos incluyendo allí la fecha y hora en la cual se inserta cada registro que proviene del sistema remoto de telemetría.

La tabla diseñada no tiene relaciones con otras entidades, puesto que el desarrollo por ahora no lo contempla, pero si es escalable para aplicaciones más robustas.

\begin{tabular}{|l|}
\hline v 0 u528746112 iot Datos \\
\# temperatura : float \\
\# humedad : float \\
1 time: timestamp \\
\hline
\end{tabular}

Fig. 24. Variables en Base de datos. Fuente: los autores.

En cuanto a la estructura del modelo de datos se tiene en cuenta dos campos de tipo flotante y un registro de tipo "timestamp" para el almacenamiento de la fecha y hora. La tabla es del tipo InnoDB, el cual actúa como mecanismo de almacenamiento de código abierto para bases de datos de MySQL [33].

Para generar el almacenamiento de los registros que provienen del sistema telemétrico se emplea el lenguaje PHP orientado a objetos que permite integrar consultas de MySQL.

De forma general se establecen las variables de conexión al motor de bases de datos y la captura de las variables por medio del método GET.

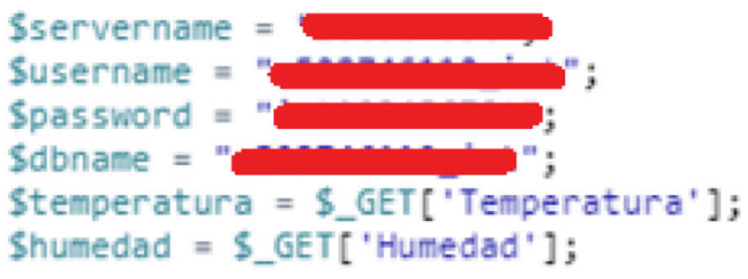

Fig. 25. Variables PHP.

Fuente: los autores.
En cuanto a los procedimientos para registrar la información capturada en la base de datos, se emplean unas sentencias en lenguaje SQL mediante el lenguaje de programación PHP orientado al desarrollo web. Se hace uso de una consulta de tipo "INSERT", la cual permite tomar valores de variables e insertarlas en los campos de una tabla establecida [34].

\$SqI = "INSERT INTO Datos (temperatura, humedad) VALUES ('\$temperatura', '\$humedad')";

Fig. 26. Consulta SQL.

Fuente: los autores.

Es así como la información captada por los sensores es enviada al servidor web y almacenada en la base de datos con el fin de ser analizada y visualizada posteriormente.

\section{INTERFAZ WEB}

La interfaz del aplicativo web permite interactuar con el usuario a través de formularios, botones, gráficos, tablas, etc. [35].

Para diseñar una interfaz amigable, clara y concreta se hace necesario hacer uso de lenguajes de diseño web como el lenguaje de estilo en cascadas CSS, lenguaje de etiquetas HTML y algún Framework que permita integrar varios aspectos en un mismo entorno de desarrollo. En el desarrollo de esta interfaz se hace uso del Framework Bootstrap, el cual es un conjunto de herramientas para diseño web, incluye plantillas para botones, formularios, tablas, menús, tipografías basadas en HTML, CSS y JavaScript [33].

Mediante el Framework de diseño web se ha realizado una interfaz responsiva, atributo muy importante a la hora de trabajar desde dispositivos móviles, esta interfaz muestra al usuario 4 módulos denominados: reportes, control, administración y seguridad.

El módulo de reportes se encarga de mostrar información y gráficos acerca de las variables temperatura y humedad relativa monitoreadas remotamente. En cuanto al módulo de control, este se encarga de permitir que el usuario pueda ejercer acciones de control ON/OFF sobre actuadores de forma remota, tales como electroválvulas de control de fluidos y solenoides para control de alimentos sólidos que serán 


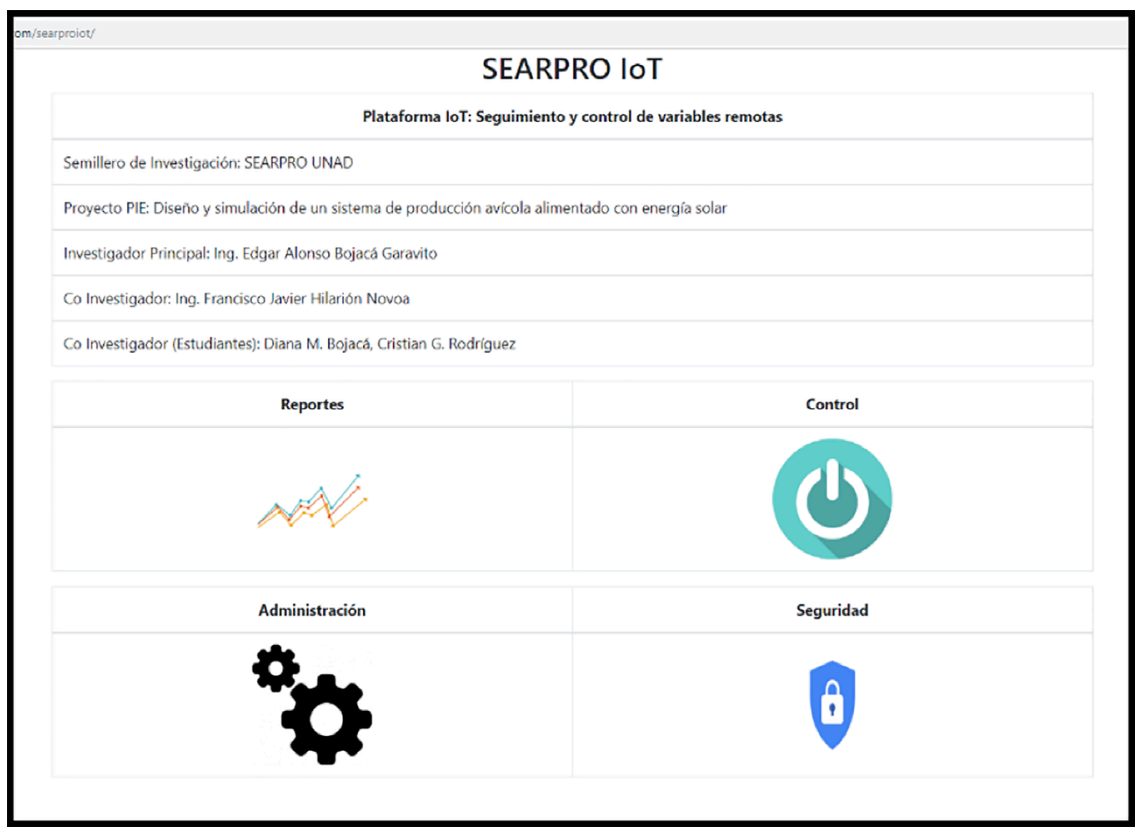

Fig. 27. Interfaz de usuario aplicativo web.

Fuente: los autores.

de gran ayuda y un respaldo de emergencia en caso de fallos del sistema automatizado de producción avícola. En cuanto al módulo de administración, desde allí es posible crear la base de datos y las tablas de forma automática sin necesidad que el usuario posea conocimientos en MySQL, además permite generar reportes en PDF sobre los valores de las variables monitoreadas. El módulo de seguridad básicamente permite establecer controles de acceso a la información y una posible asignación de roles para ciertos usuarios en cuanto el aplicativo sea más robusto.

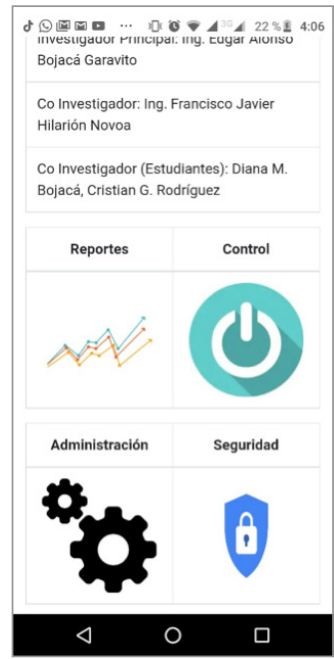

Fig. 28. Interfaz web móvil Fuente: los autores.
A continuación, se visualiza en el navegador web de un dispositivo móvil Android, el módulo de administración.

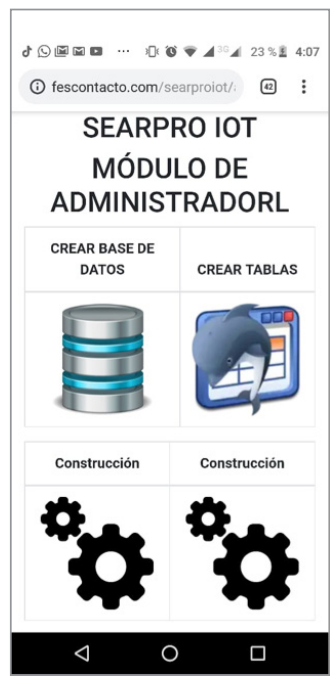

Fig. 29. Módulo de administración web móvil. Fuente: los autores.

\section{VISUALIZACIÓN DE DATOS}

La visualización de datos en el aplicativo web permite que el usuario seleccione entre un informe de valores de las variables monitoreadas, así como la 
posibilidad de generar un gráfico que represente el comportamiento dinámico en el tiempo de dichas variables adquiridas mediante la telemetría implementada [36].

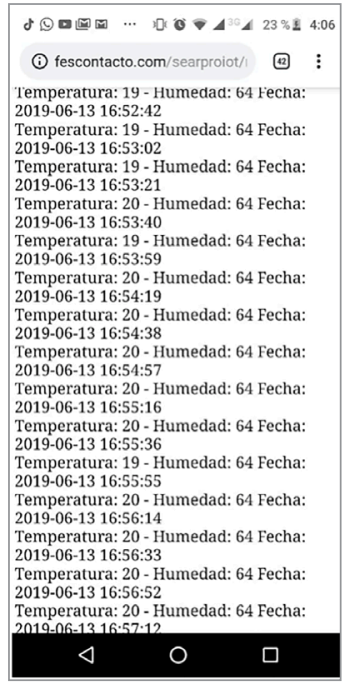

Fig. 30. Módulo reportes web.

Fuente: los autores.

Para visualizar los datos se implementa una consulta SQL automatizada sobre el lenguaje PHP, allí se selecciona los datos a mostrar, la tabla donde se alojan, los registros y su posterior despliegue sobre la interfaz.

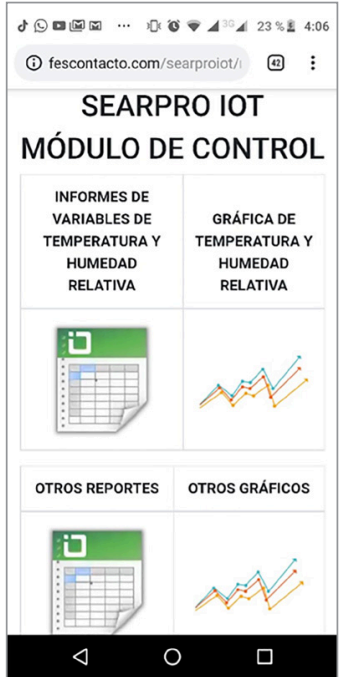

Fig. 31. Reporte de variables web.

Fuente: los autores.

Para la visualización de los gráficos se emplea la librería "Highcharts" basada en lenguajes web del lado del cliente como HTML, CSS y JavaScript ,que trabajando junto a consultas SQL permite recolectar la información, crear el gráfico y visualizar su comportamiento dinámico en el tiempo. Esta librería tiene diversas funciones, entre ellas conexión a base de datos, desconexión de base de datos, manejo de arreglos multidimensionales, consultas, y permite adaptar el tiempo para el trazo de la gráfica [37].

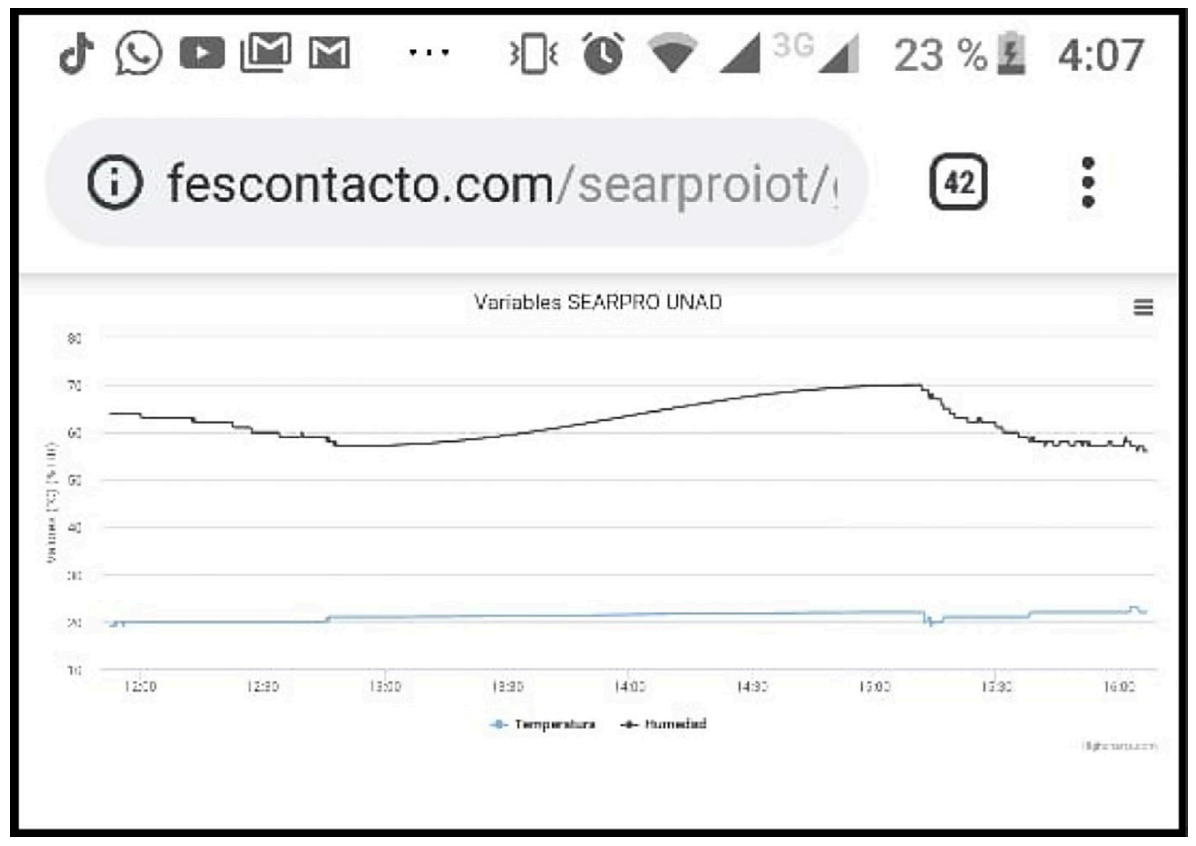

Fig. 32. Gráfico de variables web.

Fuente: los autores. 


\section{CONTROL REMOTO DE VARIABLES}

Controlar remotamente actuadores, es una tarea que requiere integrar varias disciplinas como las telecomunicaciones, la electrónica de control, electrónica de potencia, los microcontroladores, la programación móvil y la programación web. En este caso se hace énfasis en la programación web mas no en la programación móvil, ya que es un proyecto enfocado al ambiente web.

Es así como se implementa un control remoto con base en HTML para la parte gráfica y de formularios y PHP como lenguaje de procesamiento de datos [38].

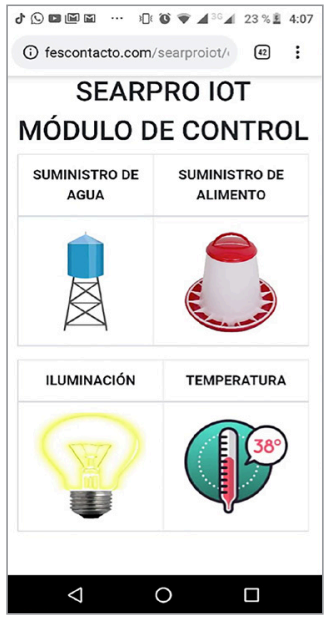

Fig. 33. Módulo de control web.

Fuente: los autores.

A continuación, se puede observar el módulo de control de agua con dos interruptores virtuales para encendido y apagado remoto.

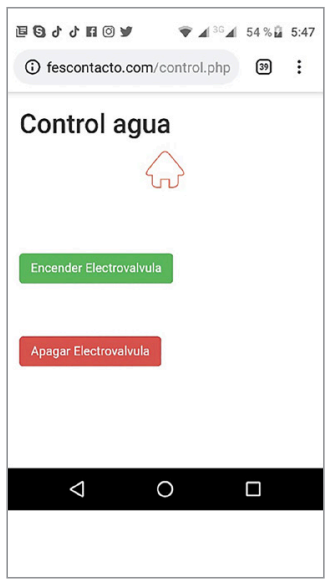

Fig. 34. Módulo control de agua web. Fuente: los autores.
A continuación, se puede observar el módulo de control de alimento con dos interruptores virtuales para encendido y apagado remoto.

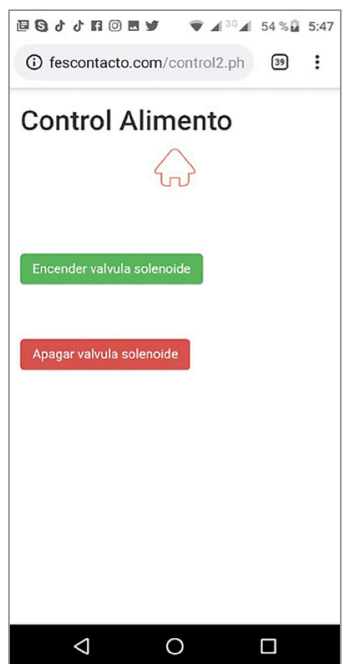

Fig. 35. Módulo de control alimento web. Fuente: los autores.

Al ejecutar las acciones de encendido o apagado se genera un archivo con el estado del proceso, el cual es almacenado en el servidor y que posteriormente es consultado por el sistema remoto para proceder a la lectura y enviar la orden al módulo de potencia para que efectúe el proceso sobre el actuador indicado y mantenerse así hasta que remotamente se ordene lo contrario [39].

La escritura del archivo se realiza mediante las funciones fopen, fwrite y fclose de PHP, que permiten trabajar con archivos planos. [40]

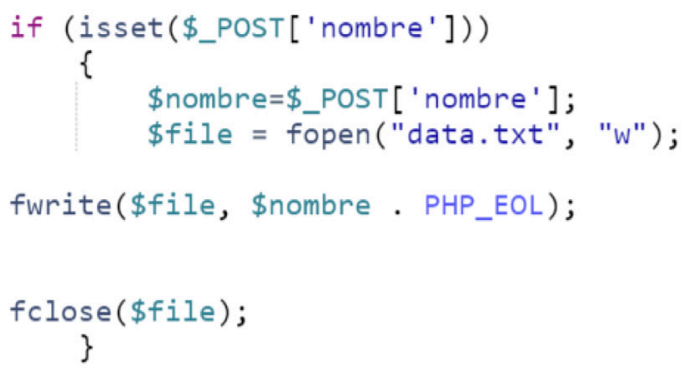

Fig. 36. Código de escritura PHP.

Fuente: los autores.

Al otro lado del sistema y precisamente en el microcontrolador NodeMCU se realiza un proceso de lectura continua sobre alguna petición originada en el servidor, y esto es posible gracias al cliente Wifi implementado con base al chip ESP8266 integrado en la tarjeta de desarrollo. 
Para este proceso de control se hace necesario establecer la configuración de pines GPIO en la tarjeta de desarrollo, los cuales se inicializan como salidas digitales en un valor lógico (0) [41].

\section{pinMode (2, OUTPUT); // Cor digitalWrite $(2,0) ; / /$ Se \\ pinMode (3, OUTPUT) ; // C digitalWrite $(3,0) ; / /$ Se}

Fig. 37. Códigos de configuración de pines GPIO.

Fuente: los autores.

Luego se realiza la conexión a la red Wifi, conexión al servidor destinado para el sitio web y revisión continua de los cambios en el archivo de texto destinado al almacenamiento de las ordenes de control.

\section{hetp-begis (- \\ int bttpCode $=$ hetp.GET (): \\ 1 (atrecede $>0)$ i \\ Serial.priacla ("Eaperando la orden"): \\ String carga $=$ btcp.getstring (): \\ Serial.printin(carga) : \\ digiealihite (2, earga. Eolne 0$)$ :}

Fig. 38. Códigos para control de puertos GPIO.

Fuente: los autores.

Posteriormente se traslada el valor leído, bien sea un uno lógico (1) o un cero lógico (0) al pin GPIO que haya sido establecido para controlar el actuador y es así como se operan remotamente las electroválvulas, solenoides u otros dispositivos.

Para el caso de este proyecto la electrónica de potencia consta de un optoacoplador y un relevador, y los actuadores están representados por una lámpara LED de 120 VAC. El manejo de la potencia es de fácil adaptación a los solenoides y electroválvulas [42].

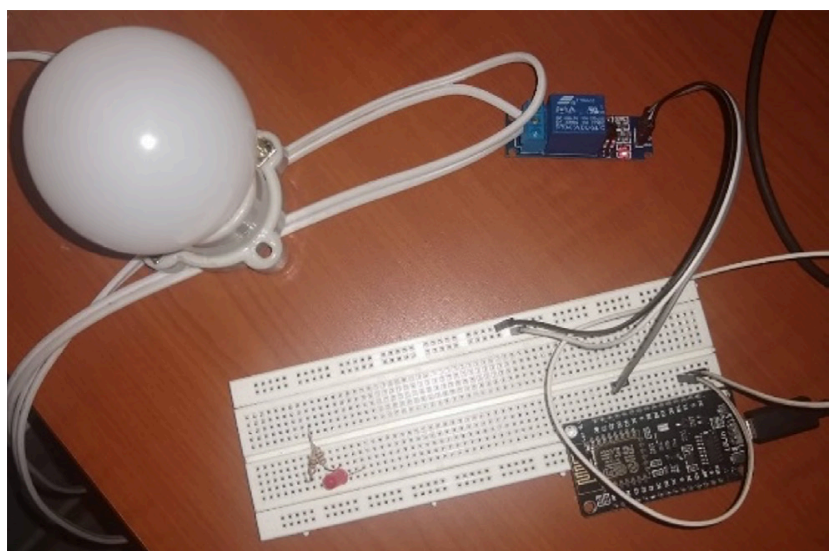

Fig. 39. Prototipo en funcionamiento.

Fuente: los autores.

\section{Simulación de la red en packet tracer}

Dentro del diseño y simulación de un sistema automatizado para producción avícola alimentado con energía solar fotovoltaica se plantea el uso de loT o internet de las cosas con el fin de realizar un proceso de monitoreo permanente de las variables que inciden en el proceso de producción avícola [43]. Inicialmente se plantea diseñar un sistema de red básico con apoyo de una serie de elementos que facilitan el monitoreo permanente de variables como:

- Temperatura.

- Humedad.

- Control de gases.

- Cierre y apertura de cortinas.

- Control del flujo de agua.

- Control de alimento de los pollos.

- Ventiladores.

- Control de iluminación.

- Control de baterías del sistema del panel solar.

La red cuenta con un sistema de direccionamiento básico en IPV4 para el proceso inicial de simulación, posiblemente durante un proceso futuro de simulación se requiera el uso del IPv6.

La red cuenta con un servidor, el cual permite almacenar la información y facilitar su consulta a través de los equipos o estaciones de trabajo, así como desde los dispositivos móviles. El servidor cuenta con un aplicativo web diseñado en PHP y HTML comunicado con la tarjeta Arduino, que captura los datos y los transmite al servidor, desde él se pueden también controlar los actuadores y recibir los reportes de la situación que presenta dentro del galpón, la siguiente figura muestra el diseño del aplicativo. 


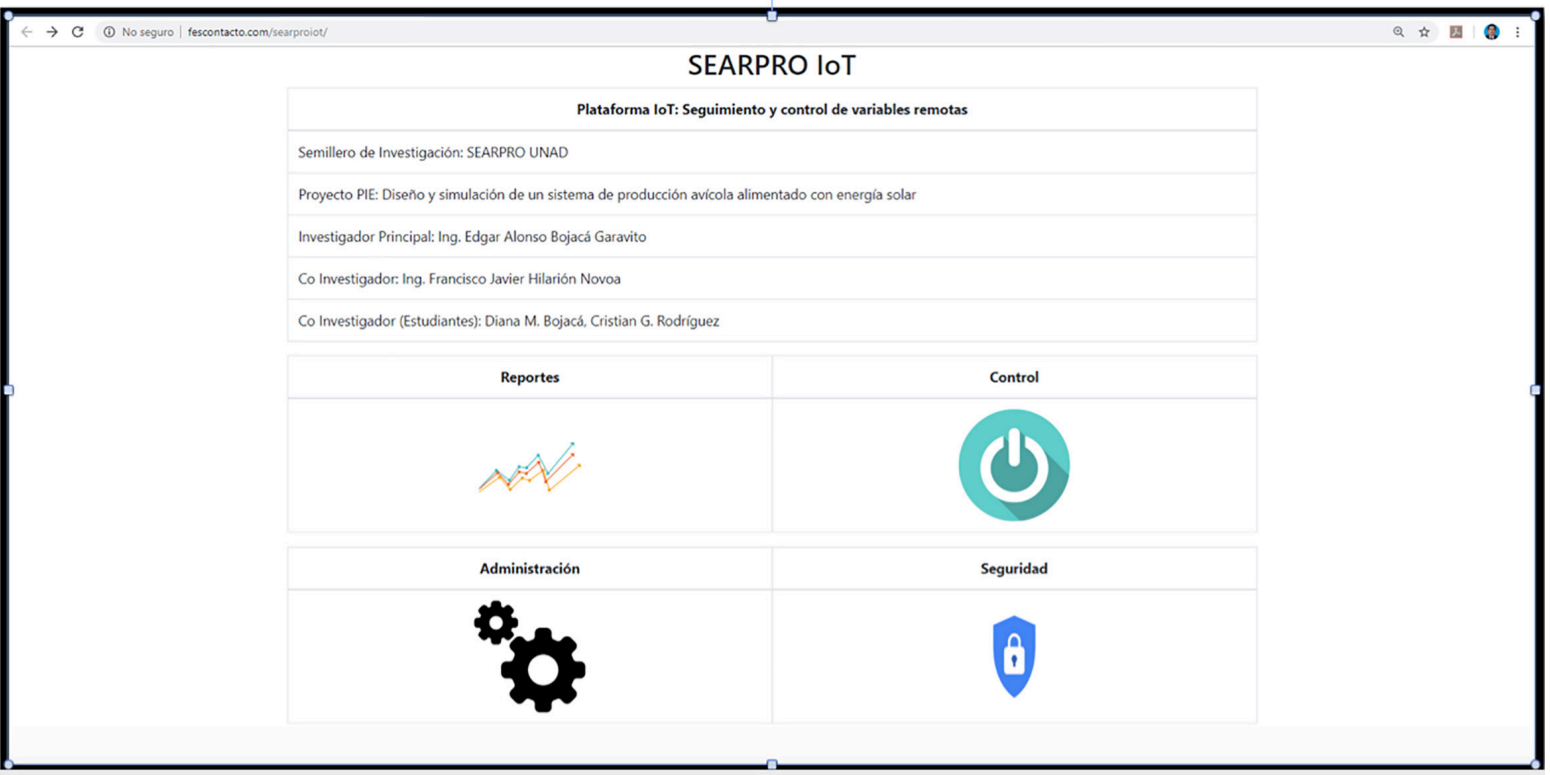

Fig. 40. Diagramas suministro de iluminación.

Fuente: los autores.

\section{ESQUEMA GENERAL DE LA RED EN PACKET TRACER 7.2}

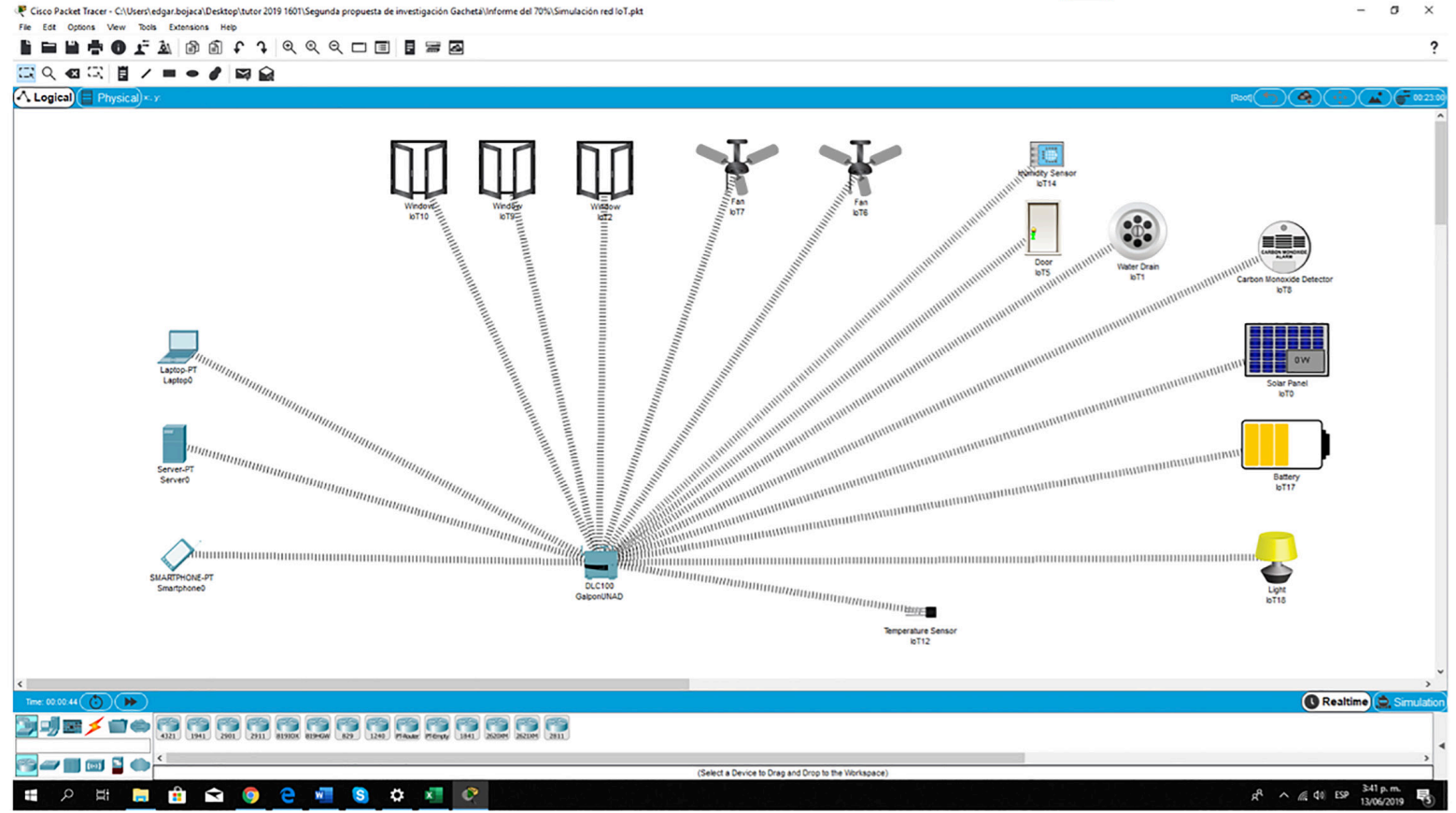

Fig. 41. Red IoT para monitoreo y control de variables.

Fuente: los autores.

El control de los servicios desde el dispositivo móvil para el caso de la simulación muestra las diferentes opciones de control del usuario a través de una interfaz gráfica [44]. 


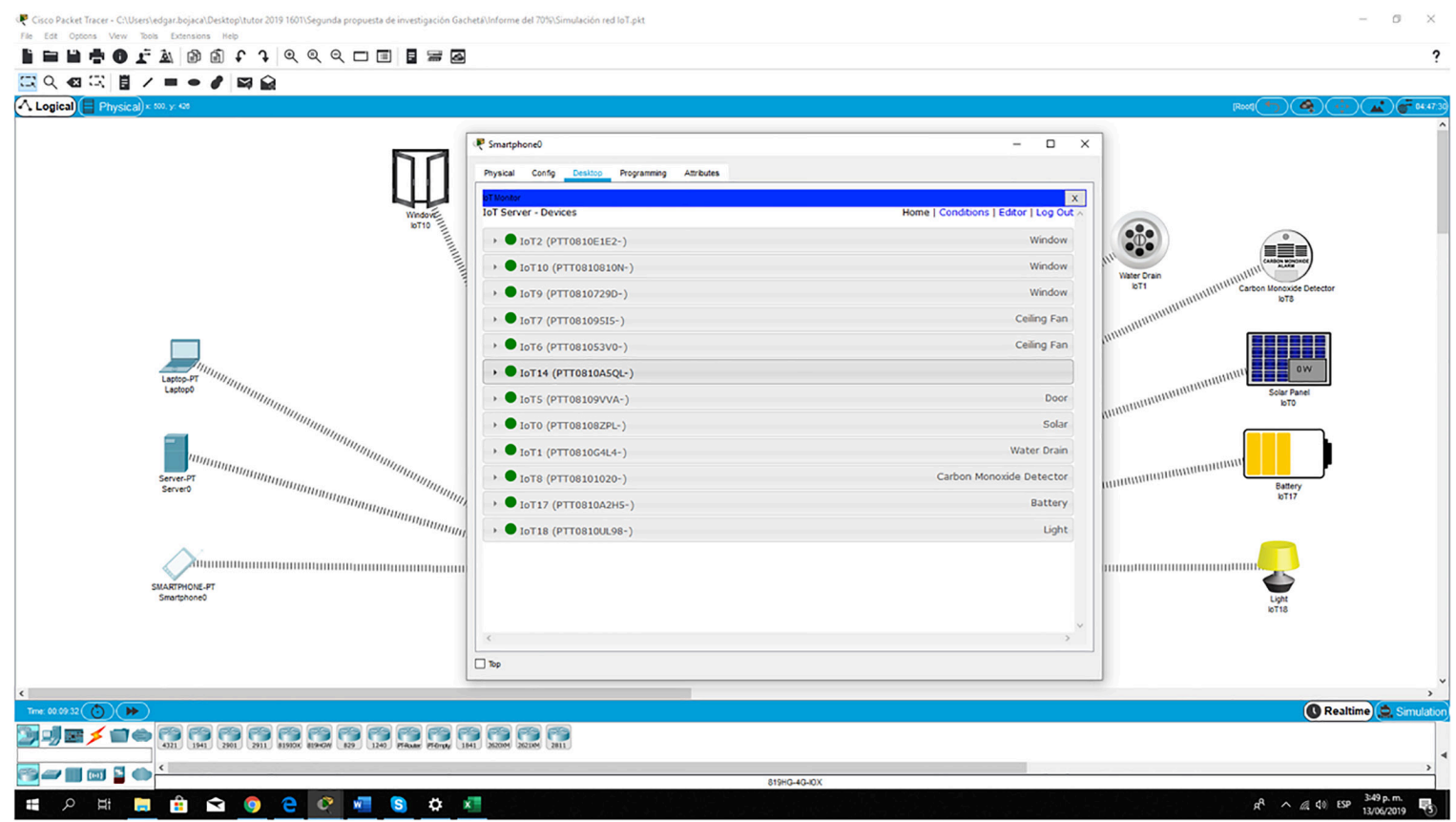

Fig. 42. Control loT de dispositivos y sensores.

Fuente: los autores.

Apertura y cierre de cortinas, la imagen muestra el control desde el dispositivo móvil abriendo y cerrando 3 cortinas creadas para el proceso de simulación, control de los ventiladores y monitoreo de las variables, como temperatura, humedad y nivel de los gases dentro del galpón. En la simulación se muestra el proceso de recepción de datos por parte de los sensores al sistema y su consulta a través del dispositivo móvil gracias a los datos alojados en el servidor.

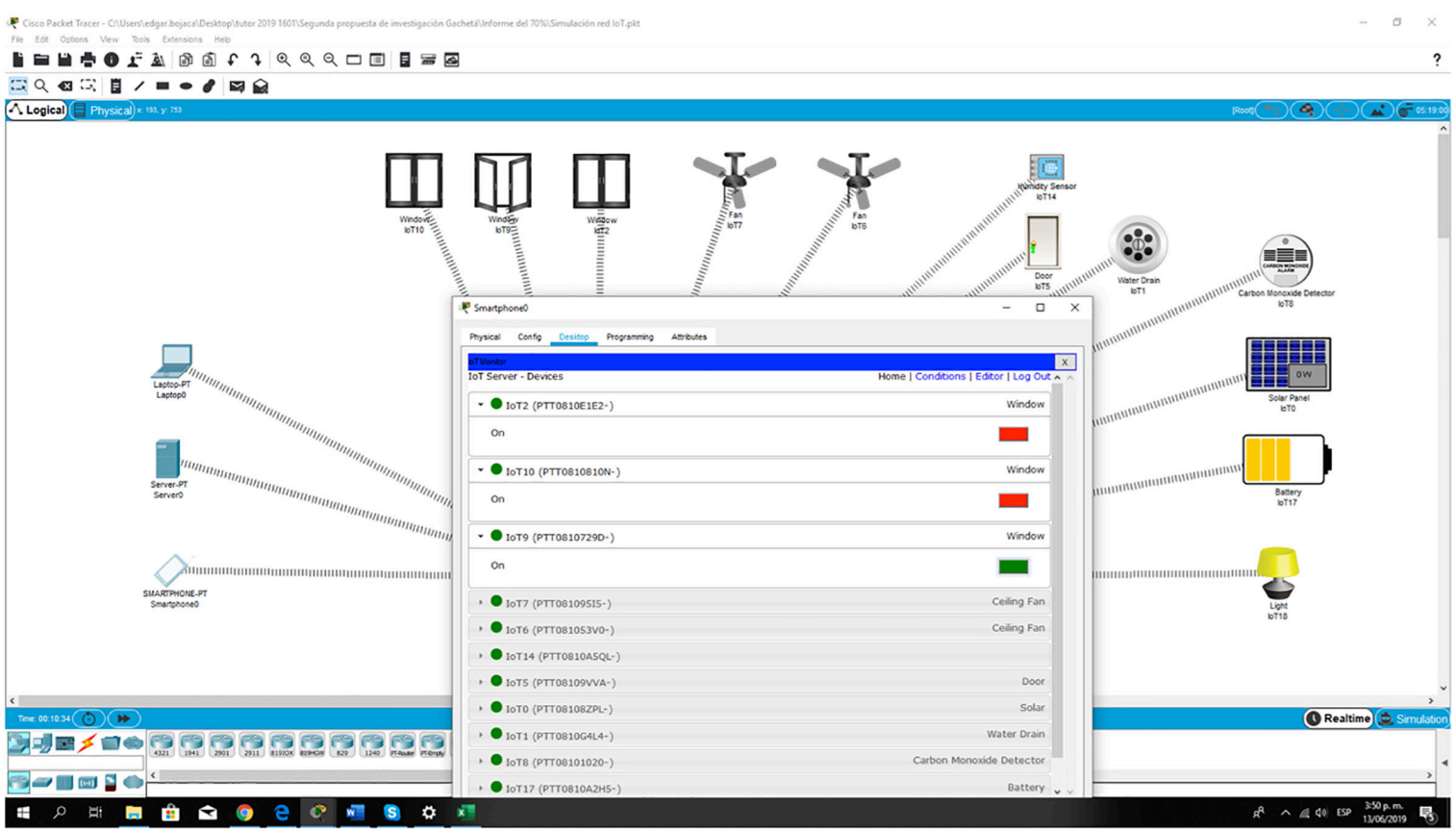

Fig. 43. Control de actuadores loT.

Fuente: los autores. 
Control de elementos como la seguridad de la puerta, la apertura del flujo del agua, los vatios generados por el panel solar, el nivel de la batería y el control de la iluminación se muestran en el dispositivo móvil una vez almacenados en el servidor, con el fin de realizar controles manuales desde el celular, se aclara que estos procesos también van a ser automatizados.

\section{Consumo eléctrico}

Ecuación de cálculo de cantidad de bombillos.

$A=$ área en $\left(\mathrm{m}^{2}\right)$

$\mathrm{L}=$ lúmenes (lux)

$\mathrm{P}=$ potencia (watts)

$\mathrm{K}=$ constante $=5$; para bombillo de 100 watt

\section{$\#$ Bombillos $=\frac{A * L}{P * k}$}

Verifiquemos el consumo de un bombillo en $\mathrm{kw} / \mathrm{h}$ y el costo del mismo para el municipio de Gachetá.

- 100 watios $=0,1 \mathrm{~kW}$

- El consumo se mide en $\mathrm{kW} \cdot \mathrm{h}$

- Costo promedio de KW/h en Gachetá: $\$ 460$

- Costo promedio para 1000 pollos: $\$ 95,220$

Pensado en reducir costos se ha verificado la instalación con bombillos led, cuyo costo es mucho menor y se mantienen los niveles de LUX, es decir la misma intensidad lumínica al mismo nivel de frecuencia de un bombillo tradicional de 100 Watts.

\section{REQUERIMIENTOS DEL SISTEMA ENERGÉTICO SOLAR}

Tras el análisis anteriormente expuesto se estima que las características del sistema solar a implementar son:

- Demanda energética para suplir las necesidades del sistema automatizado del galpón, en un día es de: 1200W [45].
- La incidencia solar para el municipio de Gachetá es $3.5 \mathrm{kw} / \mathrm{m}^{2}$.

- Factor para compensar las pérdidas 1.2.

- Potencia total del arreglo de paneles.

$$
\frac{\mathrm{FP} \times \mathrm{DE}}{\mathrm{IS}}=\frac{1.2 \times 1200}{3.5}=411 \mathrm{Wp}
$$

Por lo anterior se requiere un arreglo de paneles para desarrollar una potencia de $411 \mathrm{Wp}$.

\section{RESULTADOS Y ANÁLISIS}

Tras verificar los procesos realizados se encuentra que Arduino permite comportarse como una excelente interfaz de comunicación entre los sensores y el servidor IoT, se generan desarrollos propios del equipo investigador, los cuales dan respuesta efectiva a las necesidades dentro del proceso de producción avícola, sin embargo es necesario reforzar los sistemas con medidas adicionales que lo aíslen de factores como la humedad por exceso de agua, desechos de los animales, entre otros factores para los cuales no fueron diseñados.

Almacenar la información del ciclo de producción en bases de datos permite generar históricos de crecimiento de las aves, así como su mortalidad. Por lo anterior se pueden definir estrategias destinadas al mejoramiento de la crianza de los animales, lo anterior requiere del acompañamiento de profesionales en el área de zootecnia y veterinaria, quienes con su experiencia aportan significativamente a una futura mejora del sistema automatizado, enfocándolo en el confort del animal, lo que deriva procesos más rentables.

Se realiza un proceso de simulación efectivo con la utilización de IPv4, los procesos de implementación posiblemente requieran de configuración IPv6, por lo anterior el equipo investigador debe analizar las futuras versiones de tarjetas Arduino o similares con el fin de implementar un sistema que sea compatible con futuros desarrollos.

Luego del diseño e implementación del sistema de adquisición y procesamiento de datos se analiza en la base de datos del servidor web los datos registrados, coherencia de valores, fechas, horas 
y minutos de registro de cada dato para evitar obtener información errónea, y se establecerá el comportamiento adecuado conforme a lo planeado.

Se mantienen tiempos de registro cada 20 segundos, si bien las órdenes se realizan cada 10 segundos, hay que tener en cuenta variables como disponibilidad del canal, saturación de la red Wifi y tareas realizadas por el servidor, las cuales se están despreciando, pero los tiempos registrados son adecuados para el monitoreo de las variables establecidas.

El aplicativo web es responsivo, al realizar pruebas sobre computadores de escritorio, el comportamiento es adecuado, al hacerlo sobre un teléfono móvil se aprecia el cambio en los módulos, su presentación se adapta de forma inteligente y automática al tamaño de pantalla y tan solo al revisar el gráfico de comportamiento de las variables es necesario usar el zoom del navegador móvil con el fin de precisar cada medida, situación que es normal debido al amplio rango usado para ver el comportamiento de las variables, ya que son variables que no cambian repentinamente.

En cuanto al sistema de control remoto implementado bajo lenguajes Web y mediante la tarjeta de desarrollo NodeMCU se analiza un comportamiento preciso ya que las pruebas realizadas durante un lapso de dos horas correspondieron a lo estimado. El tiempo de respuesta para una acción de control sobre un actuador es de 1.5 segundos en promedio con un mínimo de 1 segundo y un máximo de dos segundos, lo cual teniendo en cuenta las peores condiciones de la red Wifi no tendría ninguna implicación seria en el proceso. Optimizar estos tiempos sería posible usando tarjetas de desarrollo que tengan una mayor velocidad de procesamiento y usando canales de comunicación con un ancho de banda bastante eficiente, aunque los datos que se transmiten realmente son muy pequeños.

Cabe destacar que se encontró un inconveniente en el control remoto vía web, ya que cuando los controles quedaban apagados, el usuario cerraba la aplicación web y luego volvía a abrirla, por alguna razón los actuadores se encendían, situación que generaría graves inconvenientes sobre el proceso, de igual forma sucedía cuando se suspendía el suministro de energía del sistema. Esta situación se corrigió en el código PHP que está alojado en el servidor, independizando cada archivo que contiene la orden y usando un solo archivo para cada uno de los actuadores, luego se realizaron las pruebas correspondientes siendo estas exitosas.

\section{CONCLUSIONES Y TRABAJOS FUTUROS}

Por medio de una eventual implementación de un sistema de monitoreo remoto, se pueden generar estadísticas que permitan predecir el comportamiento de las variables en el sistema de producción avícola, lo que genera mecanismos para prevenir las pérdidas, ya que la base de datos en el servidor va a guardar el registro por día, hora y minuto del cambio de cada una de las variables, así mismo el sistema permite mayores rendimientos económicos al productor avícola ya que se evita la contratación de un empleado más que monitoree las condiciones ambientales del sistema de producción y realice las tareas de alimentación e hidratación de las aves, puesto que el sistema de control remoto web permite realizarlo en un teléfono móvil desde cualquier lugar del mundo, además el sistema automático in situ del cual se hablará en otro artículo, es el encargado de automatizar la mayoría de tareas del proceso, como lo son el control de cortinas para la temperatura, iluminación y suministro individual de agua y alimentos para las aves, todo ello alimentado con energía solar fotovoltaica con el fin de evitar depender de la energía eléctrica de red convencional, el cual es un servicio que se suspende con frecuencia en la región del Guavio (Cundinamarca-Colombia) y genera pérdidas por muerte o enfermedad en las aves.

El desarrollo web es clave cuando se integra al Internet de las cosas ya que permite administrar datos, procesar información, visualizar reportes y ser interfaz de control desde dispositivos móviles.

Para realizar un desarrollo web responsivo se hace necesario integrar diversos lenguajes de programación, tanto del lado del cliente como del lado del servidor, siendo ellos PHP, SQL, JavaScript, HTML, CSS y algún Framework de diseño web como Bootstrap.

Por medio del desarrollo web del lado del cliente y del lado del servidor es posible realizar procesamiento de datos, gestión de datos, visualización de datos y control remoto de actuadores, desarrollos que pueden ser aplicados no solo a la producción avícola sino a la producción agrícola, 
y otros sectores donde sea necesario ejercer control y seguimiento a determinadas variables.

Internet de las cosas permite desarrollar sin límites, ya que es posible realizar monitoreo y control de procesos sin importar distancias, casi siempre se encuentra el mecanismo de transmisión de datos adecuado ya sea wifi, bluetooth, cable, RF, etc. Así mismo las aplicaciones son innumerables y es posible diseñar, implementar y adaptar sistemas en casi cualquier área, lo cual permite no solo mejorar rendimientos en procesos sino proyectar propuestas de valor para generar emprendimiento en las regiones.

Al realizar un monitoreo permanente del comportamiento de variables dentro del galpón se pueden realizan acciones correctivas de forma automática con el fin de optimizar el tiempo y mejorar los índices de ganancia con respecto al producto avícola.

Almacenar la información del proceso de producción permite generar un análisis de las condiciones reales del proceso, lo anterior permitirá tomar decisiones acertadas para futuras inversiones y asegurar el éxito del negocio.

Implementando el uso de energía solar se garantiza la continuidad del proceso, lo que permitirá reducir el tiempo de producción y mejorar los ingresos, lo anterior dependerá del uso adecuado y responsable de la tecnología y de que su implementación corresponda a los parámetros de diseño establecidos.

\section{REFERENCIAS}

[1] J. Jaimes-Olaya, A. Gómez, D. Álvarez, D. Soler, J. Romero, y L. Villamil, "Las enfermedades infecciosas y su importancia en el sector avícola," Rev. Med. Vet. (Bogota)., p. 13, 2010, Accessed: Nov. 21, 2019. [En Línea]. Disponible en: http://www.scielo.org.co/ $\mathrm{pdf} / \mathrm{rmv} / \mathrm{n} 20 / \mathrm{n} 20 \mathrm{a} 05 . \mathrm{pdf}$.

[2] IDEAM, “Atlas de Radiación Solar," 2015. http://atlas.ideam.gov.co/visorAtlasRadiacion.html.

[3] La Guia Cundinamarca, "La Guía Cundinamarca," 2019. https://www.laguiacundina marca.com/.
[4] O. Marcelo Ariel, Lombi; Agustín, “Aplicación de energías sustentables a la crianza de pollos," Universidad Argentina de la Empresa, 2013.

[5] M. M. Estrada-Pareja, S. M. Márquez-Girón, y L. F. R. Betancur, "Efecto de la temperatura y la humedad relativa en los parámetros productivos y la transferencia de calor en pollos de engorde," Rev. Colomb. Ciencias Pecu., vol. 20, no. 3, pp. 288-303, 2007.

[6] W. Ojeda, "CURSO POLLO DE ENGORDE: VER MANUAL," CENTRO AGRO EMPRESARIAL Y MINERO DE BOLIVAR, 2012. [En línea]. Disponible en: http://pollosantacoa.blogspot.com/ $\mathrm{p} / \mathrm{manaul}$-practico-de-pollos.html.

[7] W. Rodríguez, "Indicadores Productivos Como Herramienta Para Medir La Eficiencia Del Pollo De Engorde," Amevea-Ecuador.Org, vol. 20, p. 62, 2007, [En línea]. Disponible en: http:// amevea-ecuador.org/web_antigua/datos/ Indicadores_Productivos ING._WASHINGTON_ RODRIGUEZ.PDF\%0Ahttp: / / www.ameveaecuador.org/datos/Indicadores_Productivos ING._WASHINGTON_RODRIGUEZ.PDF.

[8] S. A. Arduino, "Arduino," Arduino LLC, 2015.

[9] Geekbot Electronics, "MQ-135 Módulo Sensor de Calidad del Aire - Geekbot Electronics," 2015. [En línea]. Disponible en: http://www. geekbotelectronics.com/producto/mq-135 -modulo-sensor-de-calidad-del-aire/.

[10] N. A. Sabuag, J. Ricohermoso, M. J. Amper, R. A. Espino, and R. Luna, "Ambient Air Quality Measurement using ATmega328 Microcontroller and MQ-135 Gas Sensor for Vehicular Emission Detection along commercial roads of Metro Manila," in [2019-MADRID] Congreso Internacional de Tecnología, Ciencia y Sociedad, 2019.

[11] M. F. Vásconez Barrera, "Diseño de un sistema de control gerencial de plantas avícolas utilizando redes de sensores inalámbricos con tecnología open hardware," Pontificia Universidad Católica del Ecuador Sede Ambato, 2015.

[12] L. del Valle, “Cómo utilizar el DHT11 para medir la temperatura y humedad con Arduino," 2016. 
[13] R. Olmos Cabrera Cotutor, B. Mohammed Al-Hadithi, and D. Raquel Cedazo León, "TRABAJO FIN DE MÁSTER Control y Monitorización de una Granja Avícola," 2013.

[14] M. M. Estrada and S. M. Márquez, “Interacción de los factores ambientales con la respuesta del comportamiento productivo en pollos de engorde," Rev. Colomb. Ciencias Pecu., vol. 18, no. 3, pp. 246-257, 2005.

[15] E. Oviedo, "El efecto de la luz en los pollos de engorde | PortalVeterinaria," 2016. .

[16] R. P. Areny, Sensores y Acondicioadores de Señal 4a. Barcelona: Marcombo, 2005.

[17] D. C. Ruiz-Ayala, C. A. Vides-Herrera, and A. Pardo-García, "Monitoreo de variables meteorológicas a través de un sistema inalámbrico de adquisición de datos," 2018.

[18] N. Tianlong, “Application of Single Bus Sensor DHT11 in Temperature Humidity Measure and Control System [J]," Microcontrollers Embed. Syst., vol. 6, p. 26, 2010, [Online]. Available: http://en.cnki.com.cn/Article_en/CJFDTOTAL-DPJY201006026.htm.

[19] H. Mora, "Sistemas de adquisición y procesamiento de datos," 2011.

[20] F. E. V. Pérez, and R. P. Areny, "Microcontroladores: Fundamentos y aplicaciones con PIC," Marcombo, 2007.

[21] A. C. Bento, “IoT: NodeMCU 12e X Arduino Uno, Results of an experimental and comparative survey," Int. J. Adv. Res. Comput. Sci. Manag. Stud., vol. 6, no. 1, 2018, [Online]. Available: https://www. researchgate. net/publication/326741893_loT_NodeMCU_12e_X_Arduino_Uno_Results_of_an_experimental_and_comparative_survey.

[22] Electronicwings, "Nodemcu Dht11 Sensor Interfacing With Nodemcu | Nodemcu," 2018. https: / / www. electronicwings.com/nodem$\mathrm{cu} /$ dht11-sensor-interfacing-with-nodemcu (accessed Aug. 29, 2019).

[23] L. K. P. Saputra, and Y. Lukito, "Implementation of air conditioning control system using REST protocol based on NodeMCU ESP8266," 2017.
[24] M. Kashyap, V. Sharma, and N. Gupta, "Taking MQTT and NodeMcu to IOT: Communication in Internet of Things," Procedia Comput. Sci., vol. 132, pp. 1611-1618, 2018, [Online]. Available: https://www.sciencedirect.com/ science/article/pii/S1877050918308585.

[25] S. H. Chiu, "Seguridad en Redes Inalámbricas 802.11," Univ. Cent. Venez., vol. 8080, p. 10, 2006, [En línea]. Disponible en: http://www. ciens.ucv.ve:8080/genasig/sites/redesmov/ archivos/Seguridad en Redes Inalambricas 802.pdf.

[26] M. Margolis, Arduino cookbook: recipes to begin, expand, and enhance your projects, 2nd ed. Massachusetts: " O'Reilly Media, Inc.," 2011.

[27] J. Candelario Elías, "Implementación de WPS en el firmware NodeMCU para el ESP8266,” 2016.

[28] C. S. Cuenca Sarango, M. Jordán, and A. Javier, "Diseño de un prototipo de cerradura electrónica conectada a una red wifi y controlada mediante una aplicación móvil, para el control automático de las puertas de los laboratorios del edificio de la FIE.," 2017.

[29] C. Mateu, "Desarrollo de aplicaciones web," 2012.

[30] N. David, A. Chima, A. Ugochukwu, and E. Obinna, "Design of a home automation system using arduino," Int. J. Sci. Eng. Res., vol. 6, no. 6, pp. 795-801, 2015, [Online]. Available: https://www.researchgate.net/publication/279179486_Design_of_a_Home_Automation_System_Using_Arduino.

[31] P. V. Sanz, Administración de sistemas gestores de bases de datos. Madrid: RA-MA Editorial, 2014.

[32] H. F. Korth, A. Silberschatz, S. Sudarshan, and F. S. Pérez, Fundamentos de bases de datos, no. QA 76.9. D32. K6718. McGraw-Hill, 1987.

[33] Á. Cobo, PHP y MySQL: Tecnología para el desarrollo de aplicaciones web. Madrid: Ediciones Díaz de Santos, 2005.

[34] M. J. Suarez-Cabal, C. de la Riva, and J. Tuya, "Populating test databases for testing SQL queries," IEEE Lat. Am. Trans., vol. 8, no. 2, pp. 164-171, 2010. 
[35] A. Fernández, “El diseño de la interfaz," Universidad de Guadalajara, 2010. https: / /www. lawebera.es/diseno-web/el-diseno-de-la-interfaz.php.

[36] M. Á. Torres Remon, Desarrollo de aplicaciones web con PHP. Barcelona: Editorial Macro, 2014.

[37] J. R. P. Ruíz, J. L. Bustos, M. C. D. I. R. Caraveo, and Á. D. M. González, "Implementación y adaptación de las librerías de HighCharts para mediciones del viento utilizando software libre," 2014.

[38] S. de Prado, "Sistema de control domótico basado en WiFi," 2017.

[39] P. K. J. Josué, C. M. O. Adrián, E. G. C. Arturo, G. L. Williams, and E. P. J. Ildefonso, "Internet de las Cosas y Sistemas Embebidos para monitorear áreas de cultivo.," 2018.
[40] M. Á. Arias, Aprende Programación Web con PHP y MySQL: 2aEdición. Vigo: IT campus Academy, 2017.

[41] J. K. Ahuluwalia, and U. Khanna, "New technique for increasing security management using Internet of Things (IOT) application," 2017.

[42] M. H. Rashid, Electrónica de potencia: circuitos, dispositivos y aplicaciones. Madrid: Pearson Educación, 2004.

[43] D. Evans, "Internet de las cosas," Cómo la próxima Evol. Internet lo cambia todo. Cisco Internet Bussiness Solut. Group-IBSG, vol. 11, no. 1, pp. 4-11, 2011.

[44] E. Sánchez, "Diseño de un sistema de control domótico basado en la plataforma Arduino," Master's thesis. Esc. Técnica Super. Ing. Informática. Univ. Politécnica Val., 2012.

[45] J. Méndez, and R. Cuervo, “Energía solar fotovoltaica," Fund. Confemetal, Madrid, pp. 27-28, 2007. 